\title{
Monge-Ampère Systems with Lagrangian Pairs
}

\author{
Goo ISHIKAWA ${ }^{\dagger}$ and Yoshinori MACHIDA $\ddagger$ \\ $\dagger$ Department of Mathematics, Hokkaido University, Sapporo 060-0810, Japan \\ E-mail: ishikawa@math.sci.hokudai.ac.jp \\ $\ddagger$ Numazu College of Technology, 3600 Ooka, Numazu-shi, Shizuoka, 410-8501, Japan \\ E-mail: machida@numazu-ct.ac.jp
}

Received April 10, 2015, in final form October 05, 2015; Published online October 10, 2015

http://dx.doi.org/10.3842/SIGMA.2015.081

\begin{abstract}
The classes of Monge-Ampère systems, decomposable and bi-decomposable Monge-Ampère systems, including equations for improper affine spheres and hypersurfaces of constant Gauss-Kronecker curvature are introduced. They are studied by the clear geometric setting of Lagrangian contact structures, based on the existence of Lagrangian pairs in contact structures. We show that the Lagrangian pair is uniquely determined by such a bi-decomposable system up to the order, if the number of independent variables $\geq 3$. We remark that, in the case of three variables, each bi-decomposable system is generated by a non-degenerate three-form in the sense of Hitchin. It is shown that several classes of homogeneous Monge-Ampère systems with Lagrangian pairs arise naturally in various geometries. Moreover we establish the upper bounds on the symmetry dimensions of decomposable and bi-decomposable Monge-Ampère systems respectively in terms of the geometric structure and we show that these estimates are sharp (Proposition 4.2 and Theorem 5.3).
\end{abstract}

Key words: Hessian Monge-Ampère equation; non-degenerate three form; bi-Legendrian fibration; Lagrangian contact structure; geometric structure; simple graded Lie algebra

2010 Mathematics Subject Classification: 58K20; 53A15; 53C42

\section{Introduction}

1.1. The second-order partial differential equation

$$
\operatorname{Hess}(f)=\operatorname{det}\left(\frac{\partial^{2} f}{\partial x_{i} \partial x_{j}}\right)_{1 \leq i, j \leq n}=c \quad(c \text { is constant, } c \neq 0),
$$

for a scalar function $f$ of $n$ real variables $x_{i}, i=1,2, \ldots, n$, describes improper (parabolic) affine hyperspheres $z=f\left(x_{1}, \ldots, x_{n}\right)$ and it plays a significant role in equi-affine geometry (see [25] for example). Similarly the equation of constant Gaussian (Gauss-Kronecker) curvature

$$
K=c \quad(c \text { is constant })
$$

for hypersurfaces is important in Riemannian geometry (see [17] for example). Note that it is written, for graphs $z=f\left(x_{1}, \ldots, x_{n}\right)$, as the equation

$$
\operatorname{Hess}(f)=(-1)^{n} c\left(1+p_{1}^{2}+\cdots+p_{n}^{2}\right)^{\frac{n+2}{2}},
$$

where $p_{i}=\frac{\partial f}{\partial x_{i}}$. Therefore the equations $\operatorname{Hess}(f)=c$ and $K=c$ are regarded as Monge-Ampère equations, and they are studied from geometric aspects in this paper. If we treat these equations in the framework of Monge-Ampère systems, then we realize that they have a specific character.

In [12], we treated improper affine spheres and constant Gaussian curvature surfaces in $\mathbf{R}^{3}$ from the view point of Monge-Ampère equations of two variables, and we analyzed the singularities of their geometric solutions. There we effectively used the direct sum decomposition of the 
standard contact structure $D \subset T \mathbf{R}^{5}$ on $\mathbf{R}^{5}$ into a pair of two Lagrangian plane fields $E_{1}, E_{2}$, namely a Lagrangian pair.

Based on the notion of Lagrangian pairs generalized to the higher-dimensional cases, namely, for contact manifolds of dimension $2 n+1$, we introduce decomposable and bi-decomposable Monge-Ampère systems with Lagrangian pairs in Section 2. A decomposable (resp. a bidecomposable) Monge-Ampère system is defined by a decomposable $n$-form (resp. a sum of two decomposable $n$-forms) which is compatible with the underlying Lagrangian pair. The class of Monge-Ampère systems with Lagrangian pairs, which is introduced in this paper, is invariant under contact transformations. If a Monge-Ampère system is isomorphic to a MongeAmpère system with a Lagrangian pair, then it is accompanied with a Lagrangian pair (see Definition 2.6). On the other hand, it is not trivial that the Lagrangian pair is uniquely associated to a given Monge-Ampère system. Then we are led to natural questions: Is the Lagrangian pair uniquely determined by the decomposable (resp. the bi-decomposable form) form? Is the Lagrangian pair recovered only from the Monge-Ampère system?

We see that Lagrangian pair is not determined by the decomposable form. Any decomposable Monge-Ampère system with Lagrangian pair $\left(E_{1}, E_{2}\right)$ is of Lagrangian type in the sense of [22], and the Lagrangian subbundle $E_{1}$ is obtained as the characteristic system of the Monge-Ampère system (see also [24] and [7, Chapter V]). However the complementary Lagrangian subbundle $E_{2}$ is not uniquely determined.

In Section 3, we show a close relation between Lagrangian pairs and bi-decomposable forms, and give an answer to the above questions by showing that a bi-decomposable Monge-Ampère system has the unique $n$-form as a local generator up to a multiplication by a non-zero function and modulo the contact form (Theorem 3.2) and that such a bi-decomposable form uniquely determines the associated Lagrangian pair $\left(E_{1}, E_{2}\right)$ uniquely, provided $n \geq 3$ (Theorem 3.8). Thus we see that any automorphism of a given Monge-Ampère system with Lagrangian pair induces an automorphism of the underlying Lagrangian pair, if $n \geq 3$. Note that Lagrangian pair is not determined by the bi-decomposable form if $n=2$ (Remark 3.11).

1.2. It follows that the study of Monge-Ampère systems with Lagrangian pairs has close relation with the theory of Takeuchi [28] on "Lagrangian contact structures".

From the viewpoint of geometric structures, the comparison of the Lagrangian contact structures and Monge-Ampère systems with Lagrangian pairs goes as follows: we treat MongeAmpère systems with Lagrangian pairs on $M=P\left(T^{*} W\right)$, the projective cotangent bundle over a manifold $W$ of dimension $n+1$ in the following two cases.

For the first case, if the base space $W$ has an affine structure, then $M=P\left(T^{*} W\right)$ has the natural Lagrangian contact structure, i.e., a Lagrangian pair, see [28]. Moreover a MongeAmpère system with the Lagrangian pair on $M$ is naturally induced, if $W$ has an equi-affine structure. Here an equi-affine structure on $W$ means that $W$ is equipped with a torsion-free linear connection and a parallel volume form on $W$, see [25]. Furthermore if $W$ is the affine flat $\mathbf{R}^{n+1}$ or the torus $T^{n+1}$, then we have the generalization of the Hessian constant equation $\operatorname{Hess}(f)=c$.

For the second case, we take a Lagrangian contact structure on $M=P\left(T^{*} W\right)$ or on the unit tangent bundle $T_{1} W$ over $W$ with the projective structure induced from a Riemannian metric on $W$. Recall that the projective structure is defined as the equivalence class of the LeviCivita connection, under the projective equivalence on torsion-free linear connections which is determined by the set of un-parametrized geodesics. Moreover we consider a Monge-Ampère system with Lagrangian pair on $M$ induced from the volume of the Riemannian metric on $W$. Furthermore if $W$ is a projectively flat Riemannian manifold, that is, one of the spaces $\mathbf{E}^{n+1}$, $S^{n+1}, H^{n+1}$ with constant curvature, we obtain the generalization of the Gaussian curvature constant equation $K=c$ as an "Euler-Lagrange" Monge-Ampère system (see Section 6 and Sections 8.2-8.5). 
We summarize those subjects as the chart:

$$
W^{n+1} \quad M^{2 n+1}=P\left(T^{*} W\right)
$$

$\left[\begin{array}{l}\text { an equi-affine structure, } \\ \text { the volume structure of a Riemannian metric }\end{array}\right] \longleftrightarrow$ a M-A system with Lagrangian pair

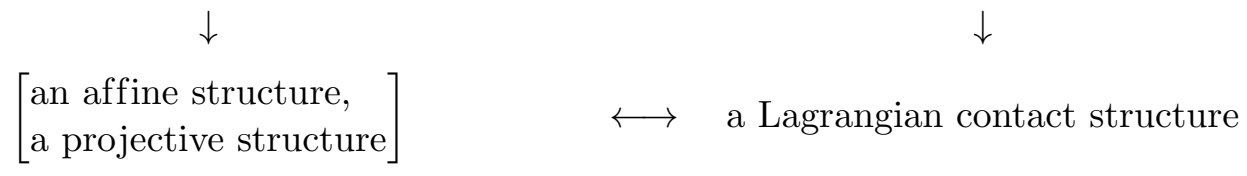

Here the lower row indicates the underlying structures and the upper row indicates the additional structures.

1.3. In Section 4 we recall the theory of Takeuchi. In Section 5, we study the symmetries of a Monge-Ampère system with a Lagrangian pair. Using the results in this paper, we show that the local automorphisms of a Monge-Ampère system with a Lagrangian pair form a finite-dimensional Lie pseudo-group, provided $n \geq 3$. We determine the maximal dimension of the automorphism pseudo-groups of the Monge-Ampère systems with flat Lagrangian pairs (Theorem 5.3).

Based on those aspects, we characterize a class of Monge-Ampère systems which includes the equations $\operatorname{Hess}(f)=c$ and $K=c, c \neq 0$. In fact, the class of Monge-Ampère equations of type

$$
\operatorname{Hess}(f)=F\left(x_{1}, \ldots, x_{n}, f(x), p_{1}, \ldots, p_{n}\right), \quad F \neq 0,
$$

is characterized and called the class of Hesse Monge-Ampère systems in Section 6. We observe that the class of Hesse Monge-Ampère systems is invariant under the contact transformations in the cases $n \geq 3$ (Proposition 6.8). For instance, the Legendre dual of the above equation is well defined and given by

$$
\operatorname{Hess}(f)=\frac{1}{F\left(p_{1}, \ldots, p_{n}, \sum_{i=1}^{n} x_{i} p_{i}-f(x), x_{1}, \ldots, x_{n}\right)} .
$$

Note that in the case $n=2, \operatorname{Hess}(f)= \pm 1$ is transformed to the Laplace equation $f_{x_{1} x_{1}}+f_{x_{2} x_{2}}=0$ or to the wave equation $f_{x_{1} x_{1}}-f_{x_{2} x_{2}}=0$. Therefore the class of Hesse Monge-Ampère systems is not invariant under the contact transformations in the case $n=2$.

In the case $n=3$, any Monge-Ampère system of the class is given by a non-degenerate threeform which is decomposed uniquely up to ordering by two decomposable forms (see [3, 11, 19, 21] and also Section 3). This fact and its generalizations are the basic reasons behind the above observation.

Further, we provide the unified picture of various subclasses of Monge-Ampère equations with significant examples in arbitrary dimensions from various geometric frameworks.

In Section 7, we introduce the general method to construct Euler-Lagrange Monge-Ampère system. We apply the method of construction to several situations and obtain several illustrative examples. In Section 8, based on the general method, we show that homogeneous Monge-Ampère systems with flat Lagrangian pairs arise in a very natural manner, in equi-affine geometry, in Euclidean geometry, in sphere geometry, in hyperbolic geometry, and moreover in Minkowski geometry. For these geometries, we construct Monge-Ampère systems with Lagrangian pairs explicitly and globally. Moreover we show that the estimate proved in Section 5 is best possible by providing the example with the maximal symmetry.

1.4. In this paper we treat, as underlying manifolds for Monge-Ampère systems, contact manifolds of dimensions $\geq 5$. We remark that 3 -dimensional contact manifolds with Lagrangian 
pairs are related to second-order ordinary differential equations with normal form. They are studied in detail in $[2,14]$.

As in [3, 4, 19, 21], Lagrangian pairs can be formulated, at least locally, on symplectic manifolds by means of reduction process. In this paper we adopt the contact framework of Monge-Ampère equations based on Lagrangian contact structures.

We remark that the paper [22] treats in detail the class of decomposable Monge-Ampère systems or Monge-Ampère systems with one decomposability, which are modeled on the MongeAmpère equation $\operatorname{Hess}(z)=0$.

We will solve the equivalence problem of Monge-Ampère systems with Lagrangian pairs in the subsequent paper.

\section{Monge-Ampère systems and Lagrangian pairs}

We start with the general definition of Monge-Ampère systems [5, 20, 23, 24]. Recall that a contact structure $D$ on a manifold $M$ is a subbundle of $T M$ of codimension one locally defined by a contact 1 -form $\theta$ by $D=\{\theta=0\}$ such that $d \theta$ is non-degenerate, that is, symplectic, on the bundle $D$. A manifold endowed with a contact structure is called a contact manifold. It is known that the dimension of a contact manifold is odd.

Let $(M, D)$ be a contact manifold of dimension $2 n+1$ with the contact structure $D \subset T M$. A Monge-Ampère system on $M$ is by definition an exterior differential system $\mathcal{M} \subset \Omega_{M}$ generated locally by a contact form $\theta$ for $D$ and an $n$-form $\omega$ on $M$ : for each point $x \in M$, there exists an open neighborhood $U$ of $x$ in $M$ such that, algebraically,

$$
\left.\mathcal{M}\right|_{U}=\langle\theta, d \theta, \omega\rangle_{\Omega_{U}}
$$

Here $\Omega_{M}$ (resp. $\Omega_{U}$ ) is the sheaf of germs of exterior differential forms on $M$ (resp. on $U$ ). In this case we call $\omega$ a local generator of the Monge-Ampère system $\mathcal{M}$ (modulo the contact ideal $\langle\theta, d \theta\rangle)$. Note that one may assume that $\omega$ is effective, i.e., $d \theta \wedge \omega \equiv 0(\bmod \theta)$. Also note that the $(n+1)$-form $d \omega$ belongs to the contact ideal locally necessarily (see [3, 4, 20]).

Let $D \subset T M$ be a vector bundle of rank $2 n$ in the tangent bundle of a manifold $M$. Recall that a conformal symplectic structure on $D$ is a reduction of the structure group of $D$ to the conformal symplectic group $\operatorname{CSp}\left(\mathbf{R}^{2 n}\right)$. If $(M, D)$ is a contact manifold of dimension $2 n+1$, then the conformal symplectic structure on $D$ is defined locally by $d \theta$ for a contact form $\theta$ which gives $D$ locally. In particular, for each point $x \in M, D_{x}$ has the symplectic structure which is determined uniquely up to a multiplication of a non-zero constant. We call a linear subspace $W \subset D_{x}$ Lagrangian if $W$ is isotropic for the conformal symplectic structure on $D_{x}$ and $\operatorname{dim}_{\mathbf{R}} W=n$. A subbundle $E \subset D$ is called a Lagrangian subbundle if $E_{x} \subset D_{x}$ is Lagrangian for any $x \in M$.

Now we define the key notion in this paper.

Definition 2.1. Let $(M, D)$ be a contact manifold. A Lagrangian pair is a pair $\left(E_{1}, E_{2}\right)$ of Lagrangian subbundles of $D$ with respect to the conformal symplectic structure on $D$ which satisfies the condition $D=E_{1} \oplus E_{2}$.

Remark 2.2. In $[6, \S 5.2]$, the notion of bi-Lagrangian structure is defined as the transverse pair of Lagrangian foliations in a symplectic manifold. Since we treat the contact case, it might be natural to use the terminology "Legendrian" instead of "Lagrangian". However we would like to use "Lagrangian" in the general cases, and to use the terminology "Legendrian" just for the integrable cases, such as "Legendrian submanifolds" and "Legendrian fibrations" (see Section 6). 
The standard example of Lagrangian pair is given as follows.

The standard example. The standard example of Lagrangian pair is given on the standard Darboux model. Consider $\mathbf{R}^{2 n+1}$ with coordinates $\left(x_{1}, \ldots, x_{n}, z, p_{1}, \ldots, p_{n}\right)$ and with the standard contact structure $D_{\text {st }}=\left\{v \in T M \mid \theta_{\text {st }}(v)=0\right\}$ defined by the standard contact form $\theta_{\text {st }}=d z-\sum_{i=1}^{n} p_{i} d x_{i}$. Then we set

$$
\begin{aligned}
& E_{1}^{\mathrm{st}}=\left\{v \in D_{\mathrm{st}} \mid d p_{1}(v)=\cdots=d p_{n}(v)=0\right\}=\left\langle\frac{\partial}{\partial x_{1}}+p_{1} \frac{\partial}{\partial z}, \ldots, \frac{\partial}{\partial x_{n}}+p_{n} \frac{\partial}{\partial z}\right\rangle, \\
& E_{2}^{\mathrm{st}}=\left\{u \in D_{\mathrm{st}} \mid d x_{1}(u)=\cdots=d x_{n}(u)=0\right\}=\left\langle\frac{\partial}{\partial p_{1}}, \ldots, \frac{\partial}{\partial p_{n}}\right\rangle .
\end{aligned}
$$

Then $\left(E_{1}^{\mathrm{st}}, E_{2}^{\mathrm{st}}\right)$ is a Lagrangian pair on $\left(\mathbf{R}^{2 n+1}, D_{\mathrm{st}}\right)$.

Note that, in [28], Takeuchi called a contact structure $D$ endowed with a Lagrangian pair $\left(E_{1}, E_{2}\right)$ a Lagrangian contact structure $\left(D ; E_{1}, E_{2}\right)$ and gave a detailed study on this geometric structure.

Moreover we consider an exterior differential system associated to a given Lagrangian pair or Lagrangian contact structure.

Definition 2.3. A Monge-Ampère system $\mathcal{M}$ is called a decomposable Monge-Ampère system with a Lagrangian pair $\left(E_{1}, E_{2}\right)$ if in a neighborhood of each point of $M$, there exists a local generator $\omega$ of $\mathcal{M}$ satisfying the following decomposing condition:

$$
i_{u} \omega=0\left(u \in E_{1}\right), \quad \text { and }\left.\quad \omega\right|_{E_{2}} \text { is a volume form on } E_{2} \text {. }
$$

Such a decomposable Monge-Ampère system of Lagrangian type is a decomposable MongeAmpère system with the characteristic system $E_{1}$ in the sense of [22]. Note that this class of Monge-Ampère equations have been introduced in [9]. Also note that decomposable MongeAmpère systems are studied from another perspective in [1] by the name of Goursat equations.

Definition 2.4. A Monge-Ampère system $\mathcal{M}$ is called a bi-decomposable Monge-Ampère system with a Lagrangian pair $\left(E_{1}, E_{2}\right)$ if, around each point of $M$, there exists a local generator $\omega$ of $\mathcal{M}$ of the form

$$
\omega=\omega_{1}-\omega_{2}
$$

by $n$-forms $\omega_{1}$ and $\omega_{2}$ satisfying the following bi-decomposing condition:

$$
\begin{aligned}
& i_{u} \omega_{1}=0 \quad\left(u \in E_{2}\right), \quad i_{v} \omega_{2}=0 \quad\left(v \in E_{1}\right), \\
& \left.\omega_{1}\right|_{E_{1}} \text { is a volume form on } E_{1}, \quad \text { and }\left.\quad \omega_{2}\right|_{E_{2}} \text { is a volume form on } E_{2} .
\end{aligned}
$$

We call such an $n$-form $\omega$ a bi-decomposable form and $\left(\omega_{1}, \omega_{2}\right)$ a bi-decomposition of $\omega$. Given a Lagrangian pair $\left(E_{1}, E_{2}\right)$ on $(M, D)$ and $n$-forms $\omega_{1}, \omega_{2}$ satisfying the bi-decomposing condition for $\left(E_{1}, E_{2}\right)$, we define a Monge-Ampère system $\mathcal{M}$ with Lagrangian pair by setting $\omega=\omega_{1}-\omega_{2}$.

Remark 2.5. An immersion $f: L \rightarrow M$ of an $n$-dimensional manifold $L$ to $M$ is called a geometric solution of a Monge-Ampère system $\mathcal{M}=\langle\theta, d \theta, \omega\rangle$ if $f^{*} \mathcal{M}=0$, namely, if $f^{*} \theta=0$, i.e., $f$ is a Legendrian immersion, and $f^{*} \omega=0$.

Any geometric solution to a decomposable (resp. bi-decomposable) Monge-Ampère system $\mathcal{M}$ with a Lagrangian pair $D=E_{1} \oplus E_{2}$ on a contact manifold $\left(M^{2 n+1}, D\right)$ has a crucial property.

In fact, an immersion $f: L \rightarrow M$ is a geometric solution of a decomposable Monge-Ampère system with a Lagrangian pair $D=E_{1} \oplus E_{2}$ if and only if $f_{*}\left(T_{p} L\right) \cap\left(E_{1}\right)_{p} \neq\{0\}$. 
For a bi-decomposable Monge-Ampère system $\mathcal{M}$ with a Lagrangian pair $D=E_{1} \oplus E_{2}$, suppose that $f: L \rightarrow M$ is a geometric solution of $\mathcal{M}$. Then, for any $p \in L$, we see that

$$
E_{1} \cap f_{*}\left(T_{p} L\right)=\{0\}, \quad \text { if and only if } \quad E_{2} \cap f_{*}\left(T_{p} L\right)=\{0\},
$$

equivalently,

$$
E_{1} \cap f_{*}\left(T_{p} L\right) \neq\{0\}, \quad \text { if and only if } \quad E_{2} \cap f_{*}\left(T_{p} L\right) \neq\{0\} .
$$

In fact, let $W$ be an $n$-plane in $D_{p}=\left(E_{1}\right)_{p} \oplus\left(E_{2}\right)_{p}$ satisfying $\left.\omega\right|_{W}=0$. The direct sum decomposition defines projections $\pi_{1}: D_{p} \rightarrow\left(E_{1}\right)_{p}$ and $\pi_{2}: D_{p} \rightarrow\left(E_{2}\right)_{p}$. Then $E_{1} \cap W=\{0\}$ if and only if $\left.\pi_{2}\right|_{W}: W \rightarrow\left(E_{2}\right)_{p}$ is an isomorphism, and $E_{2} \cap W=\{0\}$ if and only if $\left.\pi_{1}\right|_{W}: W \rightarrow$ $\left(E_{1}\right)_{p}$ is an isomorphism, respectively. For any bi-decomposition $\omega=\omega_{1}-\omega_{2}$ and for any basis $u_{1}, \ldots, u_{n}$ of $W$, we have

$$
\omega_{1}\left(\pi_{1}\left(u_{1}\right), \ldots, \pi_{1}\left(u_{n}\right)\right)=\omega_{1}\left(u_{1}, \ldots, u_{n}\right)=\omega_{2}\left(u_{1}, \ldots, u_{n}\right)=\omega_{2}\left(\pi_{2}\left(u_{1}\right), \ldots, \pi_{2}\left(u_{n}\right)\right) .
$$

Using the bi-decomposing condition again, we see that $\left.\pi_{1}\right|_{W}$ is an isomorphism if and only if the most left hand side is non-zero, and it is equivalent to the condition that $\left.\pi_{2}\right|_{W}$ is an isomorphism.

Now we are led to natural questions:

- Is the Lagrangian pair $\left(E_{1}, E_{2}\right)$ uniquely determined by a decomposable form?

- Is the Lagrangian pair $\left(E_{1}, E_{2}\right)$ uniquely determined by a bi-decomposable form?

- Is the Lagrangian pair $\left(E_{1}, E_{2}\right)$ recovered only from the Monge-Ampère system $\mathcal{M}$ ?

As is stated in Introduction, the first question is answered negatively. To answer the second and third questions, we recall the basic definitions.

Definition 2.6. Let $(M, D),\left(M^{\prime}, D^{\prime}\right)$ be contact manifolds of dimension $2 n+1$, and $\mathcal{M}, \mathcal{M}^{\prime}$ Monge-Ampère systems on contact manifolds $(M, D),\left(M^{\prime}, D^{\prime}\right)$ respectively. A diffeomorphism $\Phi: M \longrightarrow M^{\prime}$ is called an isomorphism of Monge-Ampère systems if (1) $\Phi$ is a contactomorphism, namely $\left(\Phi_{*}\right) D=D^{\prime}$, and $(2) \Phi^{*} \mathcal{M}^{\prime}=\mathcal{M}$.

Now suppose that contact manifolds $(M, D),\left(M^{\prime}, D^{\prime}\right)$ of dimension $2 n+1$ are endowed with Lagrangian pairs $\left(E_{1}, E_{2}\right),\left(E_{1}^{\prime}, E_{2}^{\prime}\right)$ respectively, namely, that the decompositions $D=E_{1} \oplus E_{2}$ and $D^{\prime}=E_{1}^{\prime} \oplus E_{2}^{\prime}$ are given. Suppose $n \geq 3$. Then, from the result in Section 3 mentioned above, we have that any isomorphism $\Phi$ of a Monge-Ampère system $\mathcal{M}$ with the Lagrangian pair $\left(E_{1}, E_{2}\right)$ and a Monge-Ampère system $\mathcal{M}^{\prime}$ with the Lagrangian pair $\left(E_{1}^{\prime}, E_{2}^{\prime}\right)$ necessarily preserves the Lagrangian pairs up to ordering, namely, $\left(\Phi_{*}\right) E_{1}=E_{1}^{\prime},\left(\Phi_{*}\right) E_{2}=E_{2}^{\prime}$ or $\left(\Phi_{*}\right) E_{1}=$ $E_{2}^{\prime},\left(\Phi_{*}\right) E_{2}=E_{1}^{\prime}$.

In the case $n=2$, a Lagrangian pair is not uniquely determined from a Monge-Ampère system and moreover, the automorphism pseudo-group may be of infinite dimension. For instance, consider the equation Hess $=-1$ which is isomorphic to the linear wave equation $f_{x_{1} x_{1}}-f_{x_{2} x_{2}}=0$ and to the equation $f_{x_{1} x_{2}}=0$. Then the last equation has infinite-dimensional automorphisms induced by diffeomorphisms $\left(x_{1}, x_{2}\right) \mapsto\left(X_{1}\left(x_{1}\right), X_{2}\left(x_{2}\right)\right)$.

\section{Lagrangian pair and bi-decomposable form}

Let $(M, D)$ be a contact manifold of dimension $2 n+1$ with a contact structure $D \subset T M$. We have defined in Section 2 the notion of bi-decomposing conditions and bi-decomposable forms on $(M, D)$. Then, first we show 
Lemma 3.1. Let $\omega$ be an $n$-form on a contact manifold $(M, D), D=\{u \in T M \mid \theta(u)=0\}$ for a local contact form $\theta$ defining $D$, and $\left(E_{1}, E_{2}\right)$ a Lagrangian pair of $D$. Assume that $\omega$ is a bi-decomposable form for $\left(E_{1}, E_{2}\right)$, and $\omega=\omega_{1}-\omega_{2}$ is any bi-decomposition of $\omega$ for $\left(E_{1}, E_{2}\right)$. Then locally there exists a coframe $\theta, \alpha_{1}, \ldots, \alpha_{n}, \beta_{1}, \ldots, \beta_{n}$ of $T^{*} M$ such that

$$
E_{1}=\left\{v \in D \mid \beta_{1}(v)=\cdots=\beta_{n}(v)=0\right\}, \quad E_{2}=\left\{u \in D \mid \alpha_{1}(u)=\cdots=\alpha_{n}(u)=0\right\},
$$

and that the $n$-forms

$$
\widetilde{\omega}_{1}=\alpha_{1} \wedge \cdots \wedge \alpha_{n}, \quad \widetilde{\omega}_{2}=\beta_{1} \wedge \cdots \wedge \beta_{n}
$$

satisfy the bi-decomposing condition for $\left(E_{1}, E_{2}\right)$ with

$$
\widetilde{\omega}_{1} \equiv \omega_{1}, \quad \widetilde{\omega}_{2} \equiv \omega_{2}, \quad \omega \equiv \widetilde{\omega}_{1}-\widetilde{\omega}_{2}
$$

up to a multiple of $\theta$.

Proof. The proof is based on the fact that the symplectic group on a finite-dimensional symplectic vector space acts transitively on the set of transversal pairs of Lagrangian subspaces.

Let $X_{1}, \ldots, X_{n}$ and $P_{1}, \ldots, P_{n}$ be local frames of $E_{1}$ and $E_{2}$ respectively. Let $R$ be the Reeb vector field for a local contact form $\theta$ defining $D$. Recall that $R$ is defined uniquely by $i_{R} \theta=1, i_{R} d \theta=0$. Consider the dual coframe $\theta, \alpha_{1}, \ldots, \alpha_{n}, \beta_{1}, \ldots, \beta_{n}$ of $T^{*} M$ to the frame $R$, $X_{1}, \ldots, X_{n}, P_{1}, \ldots, P_{n}$ of $T M$. Then we see, from the bi-decomposing condition, that there exist an $(n-1)$-form $\gamma$ and a non-vanishing function $\mu$ on $M$ such that $\omega_{1}=\mu\left(\alpha_{1} \wedge \cdots \wedge \alpha_{n}\right)+\theta \wedge \gamma$. By replacing $\alpha_{1}$ by $\frac{1}{\mu} \alpha_{1}$, we may suppose $\mu \equiv 1$. Similarly, we have $\omega_{2} \equiv \beta_{1} \wedge \cdots \wedge \beta_{n} \bmod \theta$.

Next we show that $\mathcal{M}$ has the unique local bi-decomposable generator $\omega$ modulo $\theta$.

Theorem 3.2. Let $\left(E_{1}, E_{2}\right)$ be a Lagrangian pair and $\omega, \omega^{\prime}$ be two bi-decomposable $n$-forms for the Lagrangian pair $\left(E_{1}, E_{2}\right)$ on $(M, D)$. Assume that they generate the same Monge-Ampère system

$$
\mathcal{M}=\langle\theta, d \theta, \omega\rangle=\left\langle\theta, d \theta, \omega^{\prime}\right\rangle .
$$

Then there exist locally a non-vanishing function $\mu$ and an $(n-1)$-form $\eta$ on $M$ such that $\omega^{\prime}=\mu \omega+\theta \wedge \eta$.

To show Theorem 3.2, we study, for each $x \in M$, the symplectic exterior linear algebra on the symplectic vector space $V=D_{x}$ with the symplectic form $\Theta=\left.d \theta\right|_{D_{x}}$ and with the decomposition $V=V_{1} \oplus V_{2}, V_{1}=\left(E_{1}\right)_{x}, V_{2}=\left(E_{2}\right)_{x}$, of $(V, \Theta)$ into Lagrangian subspaces.

Let $(V, \Theta)$ be a $2 n$-dimensional symplectic vector space. We say that an $n$-form $\omega \in \wedge^{n} V^{*}$ is bi-decomposable if there exist a decomposition $V=V_{1} \oplus V_{2}$ of $V$ into Lagrangian subspaces $V_{1}, V_{2}$ in $V$ and $n$-forms $\omega_{1}, \omega_{2} \in \wedge^{n} V^{*}$ such that $\omega=\omega_{1}-\omega_{2}, i_{u} \omega_{1}=0\left(u \in V_{2}\right), i_{v} \omega_{2}=0\left(v \in V_{1}\right)$, $\left.\omega_{1}\right|_{V_{1}} \neq 0,\left.\omega_{2}\right|_{V_{2}} \neq 0$. In this case $\left(\omega_{1}, \omega_{2}\right)$ is called a bi-decomposition of $\omega$. Then, similarly as the proof of Lemma 3.1, we have that there exists a basis $\left\{a_{1}, \ldots, a_{n}, b_{1}, \ldots, b_{n}\right\}$ of $V$ such that

$$
\omega_{1}=a_{1}^{*} \wedge \cdots \wedge a_{n}^{*}, \quad \omega_{2}=b_{1}^{*} \wedge \cdots \wedge b_{n}^{*},
$$

and that

$$
V_{1}=\left\langle a_{1}, \ldots, a_{n}\right\rangle, \quad V_{2}=\left\langle b_{1}, \ldots, b_{n}\right\rangle,
$$

where $\left\{a_{1}^{*}, \ldots, a_{n}^{*}, b_{1}^{*}, \ldots, b_{n}^{*}\right\}$ denotes the dual basis of $\left\{a_{1}, \ldots, a_{n}, b_{1}, \ldots, b_{n}\right\}$. Note that $V_{2}$ (resp. $\left.V_{1}\right)$ coincides with the annihilator of $a_{1}^{*}, \ldots, a_{n}^{*}\left(\operatorname{resp} . b_{1}^{*}, \ldots, b_{n}^{*}\right)$. Moreover we see that 
there exist a symplectic basis $\left\{a_{1}, \ldots, a_{n}, b_{1}, \ldots, b_{n}\right\}$ of $(V, \Theta)$ and a non-zero constants $a, b$ such that

$$
\omega=a a_{1}^{*} \wedge \cdots \wedge a_{n}^{*}-b b_{1}^{*} \wedge \cdots \wedge b_{n}^{*}
$$

If we replace $a_{1}, b_{1}$ by $(1 / b) a_{1}, b b_{1}$ respectively, so $a_{1}^{*}, b_{1}^{*}$ by $b a_{1}^{*},(1 / b) b_{1}^{*}$, and set $c=a b$, then we have the following:

Lemma 3.3. Let $\omega \in \wedge^{n} V^{*}$ be a bi-decomposable $n$-form on a $2 n$-dimensional symplectic vector space $(V, \Theta)$, and $\omega=\omega_{1}-\omega_{2}$ a bi-decomposition of $\omega$. Then there exist a symplectic basis $\left\{a_{1}, \ldots, a_{n}, b_{1}, \ldots, b_{n}\right\}$ of $(V, \Theta)$ and a non-zero constant $c$ such that

$$
\begin{aligned}
& \omega_{1}=c a_{1}^{*} \wedge \cdots \wedge a_{n}^{*}, \quad \omega_{2}=b_{1}^{*} \wedge \cdots \wedge b_{n}^{*}, \\
& \omega=c a_{1}^{*} \wedge \cdots \wedge a_{n}^{*}-b_{1}^{*} \wedge \cdots \wedge b_{n}^{*}, \quad \Theta=a_{1}^{*} \wedge b_{1}^{*}+\cdots+a_{n}^{*} \wedge b_{n}^{*} .
\end{aligned}
$$

A form $\varphi \in \wedge^{n} V^{*}$ on a symplectic vector space $(V, \Theta)$ is called effective if the interior product $i_{X_{\Theta}} \varphi=0$ for the 2-vector $i_{X_{\Theta}}$ corresponding to the 2-form $\Theta$. Note that

$$
X_{\Theta}=a_{1} \wedge b_{1}+\cdots+a_{n} \wedge b_{n}
$$

in terms of the basis in Lemma 3.3. That $\varphi \in \wedge^{n} V^{*}$ is effective if and only if the wedge $\varphi \wedge \Theta$ with the symplectic form $\Theta$ is equal to zero [4, 20]. Then we have, by Lemma 3.3:

Corollary 3.4. If $\omega \in \wedge^{n} V^{*}$ is bi-decomposable and $\omega=\omega_{1}-\omega_{2}$ is any bi-decomposition, then $\omega, \omega_{1}$ and $\omega_{2}$ are all effective.

We will apply the following basic result to our situation.

Lemma 3.5 ([20, Theorem 1.6]). Let $\omega, \omega^{\prime}$ be effective $k$-forms on a symplectic vector space $(V, \Theta),(0 \leq k \leq n)$. Suppose that, for every isotropic subspace $L \subset V$ on which $\left.\omega\right|_{L}=0$, the form $\omega^{\prime}$ also vanishes on $L,\left.\omega^{\prime}\right|_{L}=0$. Then we have $\omega^{\prime}=\mu \omega$ for some $\mu \in \mathbf{R}$.

Then we have the following:

Lemma 3.6. Let $\omega, \omega^{\prime}$ be bi-decomposable forms on $(V, \Theta)$. Suppose that $\omega^{\prime}$ is of the form $\lambda \omega+\phi \wedge \Theta$ for a scalar $\lambda$ and an $(n-2)$-form $\phi$. Then $\omega^{\prime}=\mu \omega$ for a scalar $\mu$.

Proof. By Corollary 3.4, the $n$-form $\omega$ is effective and so is the $n$-form $\omega^{\prime}$. For every Lagrangian subspace $L\left(\left.\Theta\right|_{L}=0\right)$ on which $\left.\omega\right|_{L}=0$, the form $\omega^{\prime}$ also vanishes. Therefore, by Lemma 3.5, it follows that $\omega^{\prime}=\mu \omega$ for a scalar $\mu$.

Remark 3.7. Lemma 3.6 can be shown, as an alternative proof, by applying Lefschetz isomorphism $\Theta^{2}: \wedge^{n-2} V \rightarrow \wedge^{n+2} V$. In fact, from $\omega^{\prime}-\mu \omega=\phi \wedge \Theta$ we have $\phi \wedge \Theta^{2}=0$, which implies $\phi=0$.

Proof of Theorem 3.2. Since $\omega^{\prime}$ belongs to $\mathcal{M}=\langle\theta, d \theta, \omega\rangle$, we set $\omega^{\prime}=\lambda \omega+d \theta \wedge \phi+\theta \wedge \eta$, for a function $\lambda$, an $(n-2)$-form $\phi$ and an $(n-1)$-form $\eta$ on $M$. For each $x \in M$, we have $\left.\omega^{\prime}\right|_{D_{x}}=\left.\lambda(x) \omega\right|_{D_{x}}+\left.\Theta \wedge \phi\right|_{D_{x}}$, where $\Theta=\left.d \theta\right|_{D_{x}}$. Then, by Lemma 3.6, we have $\left.\omega^{\prime}\right|_{D_{x}}=\left.\mu(x) \omega\right|_{D_{x}}$ for a scalar $\mu(x)$ depending on $x \in M$. Since $\left.\omega\right|_{\left(E_{1}\right)_{x}}$ is a volume form, we see that $\mu(x)$ is unique and of class $C^{\infty}$. Moreover there exists a $C^{\infty}(n-1)$-form $\eta$ such that $\omega^{\prime}-\mu \omega=\theta \wedge \eta$, which implies the required consequence.

Moreover we show the following basic result: 
Theorem 3.8. Assume that $n \geq 3$. Let $\mathcal{M}=\langle\theta, d \theta, \omega\rangle$ be a Monge-Ampère system with a Lagrangian pair locally generated by a bi-decomposable $n$-form $\omega$. Then $\mathcal{M}$ canonically determines the Lagrangian pair $\left(E_{1}, E_{2}\right)$. Namely, if $\left(\omega_{1}, \omega_{2}\right)$ (resp. $\left.\left(\omega_{1}^{\prime}, \omega_{2}^{\prime}\right)\right)$ is a bi-decomposition of $\omega$ for a Lagrangian pair $\left(E_{1}, E_{2}\right)$ (resp. $\left.\left(E_{1}^{\prime}, E_{2}^{\prime}\right)\right)$ enjoying the bi-decomposing condition. Then we have

$$
E_{1}^{\prime}=E_{1}, \quad E_{2}^{\prime}=E_{2}, \quad \text { or } \quad E_{1}^{\prime}=E_{2}, \quad E_{2}^{\prime}=E_{1} .
$$

Theorem 3.8 follows immediately from the following:

Proposition 3.9. Assume that $n \geq 3$. Let $(V, \Theta)$ be a $2 n$-dimensional symplectic vector space. Let $\omega=\omega_{1}-\omega_{2}$ be a bi-decomposable $n$-form for a Lagrangian pair $\left(V_{1}, V_{2}\right)$ of $V$. Then the bidecomposition $\left(\omega_{1}, \omega_{2}\right)$ of $\omega$ is unique up to ordering: If $\omega=\omega_{1}^{\prime}-\omega_{2}^{\prime}$ is another bi-decomposition of $\omega$ for another Lagrangian pair $\left(V_{1}^{\prime}, V_{2}^{\prime}\right)$ of $V$, then

$$
\begin{array}{llll}
V_{1}^{\prime}=V_{1}, & V_{2}^{\prime}=V_{2}, & \omega_{1}^{\prime}=\omega_{1}, & \omega_{2}^{\prime}=\omega_{2}, \quad \text { or } \\
V_{1}^{\prime}=V_{2}, & V_{2}^{\prime}=V_{1}, & \omega_{1}^{\prime}=-\omega_{2}, & \omega_{2}^{\prime}=-\omega_{1} .
\end{array}
$$

The bi-decomposition of $\omega$ (Proposition 3.9) is given by using the symplectic structure, based on Hitchin's result [11, Propositions 2.1, 2.2], in the case that $n$ is odd and $n \geq 3$, as follows:

Let $\omega=\omega_{1}-\omega_{2}$, with $\omega_{1}=c a_{1}^{*} \wedge \cdots \wedge a_{n}^{*}$ and $\omega_{2}=b_{1}^{*} \wedge \cdots \wedge b_{n}^{*}$, as in Lemma 3.3. Let $\epsilon=a_{1}^{*} \wedge \cdots \wedge a_{n}^{*} \wedge b_{1}^{*} \wedge \cdots b_{n}^{*}$ be the associated basis vector for $\Lambda^{2 n} V^{*}$, which is the intrinsically defined volume form by the symplectic structure on $V$. From the isomorphism $A: \Lambda^{2 n-1} V^{*} \longrightarrow$ $V \otimes \wedge^{2 n} V^{*}$ induced by the exterior product $V^{*} \otimes \wedge^{2 n-1} V^{*} \rightarrow \wedge^{2 n} V^{*}$, we define, for each $\psi \in \wedge^{n} V^{*}$, a linear transformation $K_{\psi}=K_{\psi}^{\epsilon}: V \longrightarrow V$ by $K_{\psi}(u) \epsilon=A\left(i_{u}(\psi) \wedge \psi\right)$, and put $\lambda(\psi)=\lambda_{\epsilon}(\psi)=\frac{1}{2 n} \operatorname{tr}\left(K_{\psi}^{2}\right)$.

In particular, we set $\psi=\omega$. Then we have $K_{\omega} a_{i}=-c a_{i}, K_{\omega} b_{i}=(-1)^{n+1} c b_{i}=c b_{i}$. Then we have $K_{\omega}^{2}=c^{2}$ id, therefore $\lambda(\omega)=c^{2}$. Since $K_{\omega}^{*} a_{i}^{*}=-c a_{i}^{*}, K_{\omega}^{*} b_{i}^{*}=c b_{i}^{*}$, we have

$$
K_{\omega}^{*} \omega=c(-c)^{n} a_{1}^{*} \wedge \cdots \wedge a_{n}^{*}-c^{n} b_{1}^{*} \wedge \cdots \wedge b_{n}^{*}=(-c)^{n}\left(\omega_{1}+\omega_{2}\right) .
$$

Thus, from $\omega=\omega_{1}-\omega_{2},-\frac{1}{c^{n}} K_{\omega}^{*} \omega=\omega_{1}+\omega_{2}$ and $\lambda(\omega)=c^{2}(c>0,<0)$, we see

$$
\omega_{1}=\frac{1}{2}\left(\omega \mp \lambda(\omega)^{-\frac{n}{2}} K_{\omega}^{*} \omega\right), \quad \omega_{2}=\frac{1}{2}\left(-\omega \mp \lambda(\omega)^{-\frac{n}{2}} K_{\omega}^{*} \omega\right) .
$$

Since $K_{\omega}^{*} \omega$ and $\lambda(\omega)$ are intrinsically determined from the symplectic structure on $V$, so is the decomposition of $\omega$.

To verify Proposition 3.9 in general case, we observe a fact from projective geometry of Plücker embeddings. Consider Grassmannian $\operatorname{Gr}\left(n, V^{*}\right)$ consisting of all $n$-dimensional subspaces in the $2 n$-dimensional vector space $V^{*}$, and its Plücker embedding $\operatorname{Gr}\left(n, V^{*}\right) \hookrightarrow P\left(\wedge^{n} V^{*}\right)$.

Lemma 3.10. Let $\Lambda_{1}, \Lambda_{2} \in \operatorname{Gr}\left(n, V^{*}\right)$ with $\Lambda_{1} \cap \Lambda_{2}=\{0\}$.

(1) The projective line in $P\left(\wedge^{n} V^{*}\right)$ through the two points $\Lambda_{1}, \Lambda_{2}$ does not intersect with $\operatorname{Gr}\left(n, V^{*}\right)$ at other points.

(2) Assume $n \geq 3$. Let $\Lambda_{3}$ be another point in $\operatorname{Gr}\left(n, V^{*}\right)$ different from $\Lambda_{1}, \Lambda_{2}$. Then the projective plane in $P\left(\wedge^{n} V^{*}\right)$ through the three points $\Lambda_{1}, \Lambda_{2}, \Lambda_{3}$ does not intersect with $\operatorname{Gr}\left(n, V^{*}\right)$ at other points.

Proof. Choose a basis $e_{1}, \ldots, e_{n}$ of $\Lambda_{1}$ and $e_{n+1}, \ldots, e_{2 n}$ of $\Lambda_{2}$ to form a basis of $V^{*}$. Let $\left(p_{i_{1}, i_{2}, \ldots, i_{n}}\right)$ denote Plücker coordinates of the Plücker embedding. Here $i_{1}, i_{2}, \ldots, i_{n}$ are $n$-tuple 
chosen from $\{1,2, \ldots, 2 n\}$. For any sequences $1 \leq j_{1}<j_{2}<\cdots<j_{n-1} \leq 2 n$ of $(n-1)$-letters and $1 \leq k_{1}<k_{2}<\cdots<k_{n+1} \leq 2 n$ of $(n+1)$-letters, we have the Plücker relation

$$
\sum_{\ell=1}^{n+1}(-1)^{\ell} p_{j_{1}, j_{2}, \ldots, j_{n-1}, k_{\ell}} p_{k_{1}, k_{2}, \ldots, \breve{k}_{\ell}, \ldots, k_{n+1}}=0
$$

(see $[8,10]$ for instance). To see (1), take a point $W \in \operatorname{Gr}\left(n, V^{*}\right)$ on the projective line through $\Lambda_{1}, \Lambda_{2}$. Since Plücker coordinates of $\Lambda_{1}$ (resp. $\Lambda_{2}$ ) are given by $p_{1, \ldots, n}=1$ (resp. $p_{n+1, \ldots, 2 n}=1$ ) with zero other coordinates, we have that Plücker coordinates of $W$ are given by $p_{1, \ldots, n}=\lambda, p_{n+1, \ldots, 2 n}=\mu$ for some $\lambda, \mu$, other coordinates $p_{i_{1}, \ldots, i_{n}}$ being null. Then, by applying the Plücker relation for $\left(j_{1}, \ldots, j_{n-1}\right)=(1,2, \ldots, n-1)$ and $\left(k_{1}, k_{2}, \ldots, k_{n+1}\right)=(n-1, n, \ldots, 2 n)$, we have $\lambda \mu=0$. Therefore $\lambda=0$ or $\mu=0$, namely, $W=\Lambda_{1}$ or $W=\Lambda_{2}$.

To see $(2)$, let $\bar{p}=\left(\bar{p}_{i_{1}, i_{2}, \ldots, i_{n}}\right)$ be coordinates of $\Lambda_{3}$. Then the coordinates $p=\left(p_{i_{1}, i_{2}, \ldots, i_{n}}\right)$ for a point $W$ on the plane through $\Lambda_{1}, \Lambda_{2}, \Lambda_{3}$ are given by

$$
p_{1, \ldots, n}=\lambda+\bar{p}_{1, \ldots, n}, \quad p_{n+1, \ldots, 2 n}=\mu+\bar{p}_{n+1, \ldots, 2 n}, \quad p_{i_{1}, \ldots, i_{n}}=\bar{p}_{i_{1}, \ldots, i_{n}},
$$

$\left\{i_{1}, \ldots, i_{n}\right\} \neq\{1, \ldots, n\},\{n+1, \ldots, 2 n\}$. Suppose $W \in \operatorname{Gr}\left(n, V^{*}\right), W \neq \Lambda_{1}, \Lambda_{2}, \Lambda_{3}$. Then we see that both $p$ and $\bar{p}$ satisfy the Plücker relations and that $\lambda \mu \neq 0$. Let $\left\{i_{1}, \ldots, i_{n}\right\} \neq$ $\{1, \ldots, n\},\{n+1, \ldots, 2 n\}$. Then, because $n \geq 3$, there exist $\ell, \ell^{\prime}, \ell \neq \ell^{\prime}$, such that $i_{\ell} \notin$ $\{n+1, \ldots, 2 n\}$ and $i_{\ell^{\prime}} \notin\{n+1, \ldots, 2 n\}$ or that $i_{\ell} \notin\{1, \ldots, n\}$ and $i_{\ell^{\prime}} \notin\{1, \ldots, n\}$. In the former case, we take $\left(i_{1}, \ldots, \breve{i}_{k}, \ldots, i_{n}\right),\left(i_{k}, n+1, \ldots, 2 n\right)$, and write the Plücker relation to get $\mu \cdot \bar{p}_{i_{1}, \ldots, i_{n}}=0$. In the latter case, we take $\left(i_{1}, \ldots, \breve{i}_{k}, \ldots, i_{n}\right),\left(1, \ldots, n, i_{k}\right)$ to get $\lambda \cdot \bar{p}_{i_{1}, \ldots, i_{n}}=0$. Therefore we obtain that $\bar{p}_{i_{1}, \ldots, i_{n}}=0$ for $\left\{i_{1}, \ldots, i_{n}\right\} \neq\{1, \ldots, n\},\{n+1, \ldots, 2 n\}$. This means that $\Lambda_{3}$ lies on the projective line through $\Lambda_{1}, \Lambda_{2}$, which contradicts (1).

Proof of Proposition 3.9. Set $\omega_{1}-\omega_{2}=\omega_{1}^{\prime}-\omega_{2}^{\prime}$. Then $\omega_{1}-\omega_{2}-\omega_{1}^{\prime}$ is equal to a decomposable form $-\omega_{2}^{\prime}$. Let $\Lambda_{1}, \Lambda_{2}, \Lambda_{1}^{\prime}, \Lambda_{2}^{\prime}$ be points in $\operatorname{Gr}\left(n, V^{*}\right) \subset P\left(\wedge^{n} V^{*}\right)$ corresponding to $\omega_{1}, \omega_{2}, \omega_{1}^{\prime}$, $\omega_{2}^{\prime}$ respectively. Then the projective plane through $\Lambda_{1}, \Lambda_{2}, \Lambda_{1}^{\prime}$ intersects with $\operatorname{Gr}\left(n, V^{*}\right)$ also at $\Lambda_{2}^{\prime}$. Note that $\Lambda_{1}^{\prime} \neq \Lambda_{2}^{\prime}$ as well as $\Lambda_{1} \neq \Lambda_{2}$. Suppose $\Lambda_{1}^{\prime} \neq \Lambda_{1}, \Lambda_{2}$. By Lemma 3.10(2), we have $\Lambda_{2}^{\prime}=\Lambda_{1}$ or $\Lambda_{2}^{\prime}=\Lambda_{2}$. Then we have that $\Lambda_{1}^{\prime}$ lies on the line through $\Lambda_{1}, \Lambda_{2}$. By Lemma 3.10(1), we conclude that $\Lambda_{1}^{\prime}=\Lambda_{1}$ or $\Lambda_{1}^{\prime}=\Lambda_{2}$. If $\omega_{1}^{\prime}=\lambda \omega_{1}$ for some $\lambda \neq 0$, then $V_{2}^{\prime}=V_{2}$ as the annihilator of $\omega_{1}$ or $\omega_{1}^{\prime}$. If $\omega_{1}^{\prime}=\mu \omega_{2}$ for some $\mu \neq 0$, then we have $V_{2}^{\prime}=V_{1}$. By the symmetric argument, we obtain also that $V_{1}^{\prime}=V_{1}$ or $V_{1}^{\prime}=V_{2}$. Thus we have $V_{1}^{\prime}=V_{1}, V_{2}^{\prime}=V_{2}$ or $V_{1}^{\prime}=V_{2}$, $V_{2}^{\prime}=V_{1}$. Assume $V_{1}^{\prime}=V_{1}, V_{2}^{\prime}=V_{2}$, then, restricting $\omega$ to $V=V_{1} \oplus V_{2}$, we have $\omega_{1}^{\prime}=\omega_{1}$, $\omega_{2}^{\prime}=\omega_{2}$. Assume $V_{1}^{\prime}=V_{2}, V_{2}^{\prime}=V_{1}$, then we have $\omega_{1}^{\prime}=-\omega_{2}, \omega_{2}^{\prime}=-\omega_{1}$.

Remark 3.11. If $n=2$, that is, $M$ is of dimension 5, then Theorem 3.8 does not hold. In fact, consider $M=\mathbf{R}^{5}$ with coordinates $(x, y, z, p, q)$ and with the contact form $\theta=d z-p d x-q d y$. Take a 2 -form

$$
\omega=d x \wedge d y-d p \wedge d q
$$

Then decomposable 2 -forms $\omega_{1}=d x \wedge d y, \omega_{2}=d p \wedge d q$ satisfy $\omega=\omega_{1}-\omega_{2}$ and the bi-decomposing condition for the Lagrangian pair given by

$$
\begin{aligned}
& E_{1}=\left\{v \in T \mathbf{R}^{5} \mid \theta(v)=d p(v)=d q(v)=0\right\}=\left\langle\frac{\partial}{\partial x}+p \frac{\partial}{\partial z}, \frac{\partial}{\partial y}+q \frac{\partial}{\partial z}\right\rangle, \\
& E_{2}=\left\{u \in T \mathbf{R}^{5} \mid \theta(u)=d x(u)=d y(u)=0\right\}=\left\langle\frac{\partial}{\partial p}, \frac{\partial}{\partial q}\right\rangle .
\end{aligned}
$$


Then we can find other decomposable 2-forms $\omega_{1}^{\prime}=d(x+p) \wedge d y, \omega_{2}^{\prime}=d p \wedge d(y+q)$ satisfying $\omega=\omega_{1}^{\prime}-\omega_{2}^{\prime}$ and the bi-decomposing condition for another Lagrangian pair given by

$$
\begin{aligned}
& E_{1}^{\prime}=\left\{v \in T \mathbf{R}^{5} \mid \theta(v)=d p(v)=d(y+q)(v)=0\right\}=\left\langle\frac{\partial}{\partial x}+p \frac{\partial}{\partial z}, \frac{\partial}{\partial y}+q \frac{\partial}{\partial z}-\frac{\partial}{\partial q}\right\rangle, \\
& E_{2}^{\prime}=\left\{u \in T \mathbf{R}^{5} \mid \theta(u)=d(x+p)(u)=d y(u)=0\right\}=\left\langle\frac{\partial}{\partial x}+p \frac{\partial}{\partial z}-\frac{\partial}{\partial p}, \frac{\partial}{\partial q}\right\rangle .
\end{aligned}
$$

Lemma 3.10(2) does not hold in the case $n=2$, because $\operatorname{Gr}\left(2, \mathbf{R}^{4}\right) \hookrightarrow P\left(\wedge^{2}\left(\mathbf{R}^{4}\right)\right)=P^{5}$ is a hypersurface and a projective plane intersects with $\operatorname{Gr}\left(2, \mathbf{R}^{4}\right)$ in infinite points (a planer curve).

From the above-mentioned propositions, it follows that, in the case $n \geq 3$, the notion of a bi-decomposable Monge-Ampère system with a Lagrangian pair $\left(E_{1}, E_{2}\right)$ is nothing but the notion of a Monge-Ampère system generated by a bi-decomposable form $\omega=\omega_{1}-\omega_{2}$.

\section{Lagrangian contact structures}

Let us recall Takeuchi's paper [28] for Lagrangian contact structures. A contact structure with a Lagrangian pair is called a Lagrangian contact structures in [28]. A typical example of Lagrangian contact structures is the projective cotangent vector bundle $M=P\left(T^{*} W\right)$ of an $(n+1)$-dimensional manifold $W$ with an affine structure (a torsion-free linear connection) or a projective structure (a projective equivalence class of torsion-free linear connections). For the canonical contact structure $D$ on $M$, we take as a Lagrangian pair horizontal and vertical vector bundles (cf. Example 6.3). In [28], it is given the description of Cartan connections on $P\left(T^{*} W\right)$ associated to Lagrangian contact structures and the equivalence of the vanishing of the curvature of Cartan connection and the projective flatness of $W$.

The flat model, which is a homogeneous space qualified as a model for a Cartan connection, with Lagrangian contact structure is the projective cotangent bundle $P\left(T^{*} P^{n+1}\right)$ of the $(n+1)$ dimensional projective space $P^{n+1}=P\left(\mathbf{R}^{n+2}\right)$.

Put $G=\operatorname{PGL}(n+2, \mathbf{R})=\operatorname{GL}(n+2, \mathbf{R}) / C\left(C\right.$ is the center, $\left.C=\mathbf{R}^{\times} \cdot I_{n+2}\right)$, and $\mathfrak{g}=\operatorname{Lie} G \cong$ $\mathfrak{s l}(n+2, \mathbf{R})$. The Lie algebra $\mathfrak{g}$ has a structure of a simple graded Lie algebra (GLA) of second kind as follows:

$$
\begin{aligned}
& \mathfrak{g}= \mathfrak{s l}(n+2, \mathbf{R})=\mathfrak{g}_{-2} \oplus \mathfrak{g}_{-1} \oplus \mathfrak{g}_{0} \oplus \mathfrak{g}_{1} \oplus \mathfrak{g}_{2} \\
&=\left\{\left(\begin{array}{ccc}
0 & 0 & 0 \\
0 & \mathrm{O}_{n} & 0 \\
a & 0 & 0
\end{array}\right)\right\} \oplus\left\{\left(\begin{array}{ccc}
0 & 0 & 0 \\
b_{1} & \mathrm{O}_{n} & 0 \\
0 & { }^{t} b_{2} & 0
\end{array}\right)\right\} \oplus\left\{\left(\begin{array}{ccc}
\alpha & 0 & 0 \\
0 & A & 0 \\
0 & 0 & \beta
\end{array}\right)\right\} \\
& \oplus\left\{\left(\begin{array}{ccc}
0 & { }^{t} c_{1} & 0 \\
0 & \mathrm{O}_{n} & c_{2} \\
0 & 0 & 0
\end{array}\right)\right\} \oplus\left\{\left(\begin{array}{ccc}
0 & 0 & d \\
0 & \mathrm{O}_{n} & 0 \\
0 & 0 & 0
\end{array}\right)\right\}, \\
&\left(a, d, \alpha, \beta \in \mathbf{R}, b_{1}, b_{2}, c_{1}, c_{2} \in \mathbf{R}^{n}, A \in \mathfrak{g l}(n, \mathbf{R}) ; \alpha+\beta+\operatorname{tr} A=0\right), \quad\left[\mathfrak{g}_{p}, \mathfrak{g}_{q}\right] \subset \mathfrak{g}_{p+q} .
\end{aligned}
$$

Put

$$
\mathfrak{m}=\mathfrak{g}_{-2} \oplus \mathfrak{g}_{-1}
$$

then $\mathfrak{m}$ is a fundamental GLA of contact type, i.e., Heisenberg algebra. Put

$$
\mathfrak{g}^{\prime}=\mathfrak{g}_{0} \oplus \mathfrak{g}_{1} \oplus \mathfrak{g}_{2}
$$


Let $G^{\prime}$ be the Lie subgroup of $G=\operatorname{PGL}(n+2, \mathbf{R})$ defined by

$$
G^{\prime}=P\left\{\left(\begin{array}{lll}
* & * & * \\
0 & * & * \\
0 & 0 & *
\end{array}\right) \in \mathrm{GL}(n+2, \mathbf{R})\right\} .
$$

Then the Lie algebra of $G^{\prime}$ is given by $\mathfrak{g}^{\prime}$. Note that $\operatorname{dim} \mathfrak{g}^{\prime}=n^{2}+2 n+2$.

The group $G$ transitively acts on the flag manifold

$$
P\left(T^{*} P^{n+1}\right)=\left\{V_{1} \subset V_{n+1} \subset \mathbf{R}^{n+2} \mid \operatorname{dim} V_{1}=1, \operatorname{dim} V_{n+1}=n+1\right\} \subset P^{n+1} \times P^{n+1 *} .
$$

Then $G^{\prime}$ is the isotropy group of $\left(V_{1}, V_{n+1}\right)=\left(\left\langle e_{0}\right\rangle,\left\langle e_{0}, \ldots, e_{n}\right\rangle\right)$ for the standard basis $e_{0}, e_{1}, \ldots$, $e_{n}, e_{n+1}$ of $\mathbf{R}^{n+1}$. Therefore we have

$$
G / G^{\prime} \cong P\left(T^{*} P^{n+1}\right) \quad\left(\cong P\left(T^{*} P^{n+1 *}\right)\right) .
$$

Note that $\mathfrak{g}_{-1} \subset \mathfrak{m}=T_{o}\left(G / G^{\prime}\right)$, where $o=G^{\prime}$ is the origin, defines the contact structure $D$ on $G / G^{\prime}$ which corresponds to the canonical contact structure on $P\left(T^{*} P^{n+1}\right)$ via the above diffeomorphism (cf. [12]).

Next, we consider

$$
\mathfrak{e}^{1}=\left\{\left(\begin{array}{ccc}
0 & 0 & 0 \\
b_{1} & \mathrm{O}_{n} & 0 \\
0 & 0 & 0
\end{array}\right)\right\}, \mathfrak{e}^{2}=\left\{\left(\begin{array}{ccc}
0 & 0 & 0 \\
0 & \mathrm{O}_{n} & 0 \\
0 & { }^{t} b_{2} & 0
\end{array}\right)\right\} .
$$

Then we have

$$
\begin{aligned}
& \mathfrak{g}_{-1}=\mathfrak{e}^{1} \oplus \mathfrak{e}^{2}, \quad\left[\mathfrak{e}^{1}, \mathfrak{e}^{1}\right]=\left[\mathfrak{e}^{2}, \mathfrak{e}^{2}\right]=0, \quad \mathfrak{g}_{-2}=\left[\mathfrak{e}^{1}, \mathfrak{e}^{2}\right], \\
& {\left[\mathfrak{g}_{0}, \mathfrak{e}^{1}\right] \subset \mathfrak{e}^{1}, \quad\left[\mathfrak{g}_{0}, \mathfrak{e}^{2}\right] \subset \mathfrak{e}^{2} .}
\end{aligned}
$$

Denote by $E_{i j} \in \mathfrak{g l}(n+2, \mathbf{R})$ the matrix unit of $(i, j)$ component. Then we put

$$
\begin{aligned}
& \gamma=E_{n+2,1} \in \mathfrak{g}_{-2}, \\
& e_{i}=E_{i+1,1} \in \mathfrak{e}^{1} \subset \mathfrak{g}_{-1}, \quad f_{i}=E_{n+2, i+1} \in \mathfrak{e}^{2} \subset \mathfrak{g}_{-1} \quad(1 \leq i \leq n) .
\end{aligned}
$$

We set

$$
[X, Y]=-A(X, Y) \gamma \quad\left(X, Y \in \mathfrak{g}_{-1}\right) .
$$

It follows that

$$
A\left(e_{i}, f_{j}\right)=\delta_{i j}, \quad A\left(e_{i}, e_{j}\right)=0, \quad A\left(f_{i}, f_{j}\right)=0 \quad(1 \leq i, j \leq n) .
$$

Therefore we have that $A$ is a symplectic form. Then $\mathfrak{g}_{-1}$ becomes a symplectic vector space with respect to $A$, and $e_{1}, \ldots, e_{n}, f_{1}, \ldots, f_{n}$ form a symplectic basis of the symplectic vector space $\left(\mathfrak{g}_{-1}, A\right)$. Moreover $\mathfrak{e}^{1}, \mathfrak{e}^{2}$ form a Lagrangian pair of $\left(\mathfrak{g}_{-1}, A\right)$.

Moreover, put

$$
\mathfrak{a}^{1}=\mathfrak{e}^{1}+\mathfrak{g}^{\prime}, \quad \mathfrak{a}^{2}=\mathfrak{e}^{2}+\mathfrak{g}^{\prime} .
$$

Then we easily verify that

$$
\operatorname{Ad}\left(G^{\prime}\right) \mathfrak{a}^{1}=\mathfrak{a}^{1}, \quad \operatorname{Ad}\left(G^{\prime}\right) \mathfrak{a}^{2}=\mathfrak{a}^{2}, \quad \mathfrak{a}^{1} \cap \mathfrak{a}^{2}=\mathfrak{g}^{\prime} .
$$


Thus $\mathfrak{a}^{1}$ and $\mathfrak{a}^{2}$ induce invariant differential systems $E_{1}$ and $E_{2}$ on $G / G^{\prime}$ respectively. The pair $\left(E_{1}, E_{2}\right)$ forms a Lagrangian pair of $\left(G / G^{\prime}, D\right)$ and thus that of the standard contact structure $D$ on $P\left(T^{*} P^{n+1}\right)$. Moreover both $E_{1}$ and $E_{2}$ are completely integrable. It follows that $P\left(T^{*} P^{n+1}\right) / \mathcal{E}_{2} \cong P^{n+1}, P\left(T^{*} P^{n+1}\right) / \mathcal{E}_{1} \cong P^{n+1^{*}}$, where $\mathcal{E}_{1}, \mathcal{E}_{2}$ are the foliations induced by $E_{1}, E_{2}$ respectively.

For the linear isotropy representation $\rho: G^{\prime} \longrightarrow \mathrm{GL}(\mathfrak{m})$ at the origin $o=G^{\prime}$ in $G / G^{\prime}$, we have, with respect to the basis $\left\{\gamma, e_{1}, \ldots, e_{n}, f_{1}, \ldots, f_{n}\right\}$ in $\mathfrak{m}$,

$$
\tilde{G}=\rho\left(G^{\prime}\right)=\left\{\left(\begin{array}{ccc}
a & 0 & 0 \\
b_{1} & A & \mathrm{O}_{n} \\
b_{2} & \mathrm{O}_{n} & a^{t} A^{-1}
\end{array}\right) \mid a \in \mathbf{R}^{*}, A \in \mathrm{GL}(n, \mathbf{R}), b_{1}, b_{2} \in \mathbf{R}^{n}\right\} .
$$

Then the $\tilde{G}$-structures of type $\mathfrak{m}$ are in bijective correspondence with the Lagrangian contact structures [28, Theorem 5.1]. Note that $\tilde{G}$-structure is of infinite type [16, Chapter I]. However $\mathfrak{g}$ is the prolongation of $\left(\mathfrak{m}, \mathfrak{g}_{0}\right)$ in the sense of Tanaka [30, 31]. Moreover $\tilde{G}=G_{0}^{\#}$ in the notation of [29]. Thus, by the finiteness theorem of Tanaka [29, Corollary 2], we have:

Proposition 4.1. Let $(M, D)$ be a contact manifold of dimension $2 n+1$ with a Lagrangian pair $\left(E_{1}, E_{2}\right)$. Then the automorphism pseudo-group of all compatible diffeomorphisms on $M$ as a Lagrangian contact structure is of finite type, that is to say, it is a finite-dimensional Lie pseudo-group. The maximum dimension of the automorphism pseudo-groups, fixing $n$, is given by $\operatorname{dim} \mathfrak{s l}(n+2, \mathbf{R})=(n+2)^{2}-1$ which the flat model attains.

Moreover, using a result in [22], we have:

Proposition 4.2. The equivalence class of a decomposable Monge-Ampère system with a Lagrangian pair $\left(E_{1}, E_{2}\right)$ on a contact manifold $(M, D)$ is uniquely determined by the Lagrangian contact structure $\left(D, E_{1}, E_{2}\right)$. Therefore the maximal dimension of the automorphism pseudogroups of decomposable Monge-Ampère systems on $\left(M^{2 n+1}, D\right)$ is equal to $(n+2)^{2}-1$. The maximum is attained by the decomposable Monge-Ampère system Hess $=0$ on the flat model on $M=P T^{*}\left(P^{n+1}\right)$.

In fact it is given the following result in [22], which implies Proposition 4.2:

Lemma 4.3 ([22, Proposition 2.1]). Let $(V, \Theta)$ be a symplectic vector space of dimension $2 n$. For given two non-zero decomposable n-covectors $\omega=\beta_{1} \wedge \cdots \wedge \beta_{n}, \omega^{\prime}=\beta_{1}^{\prime} \wedge \cdots \wedge \beta_{n}^{\prime}$ with $\beta_{i}, \beta_{i}^{\prime} \in V^{*}$, we have $\omega^{\prime}=\lambda \omega+\phi \wedge \Theta$ for a nonzero scalar $\lambda$ and a $(n-2)$-covector $\phi$, if and only if, the annihilator of $\beta_{1}, \ldots, \beta_{n}$ in $V$ and the annihilator of $\beta_{1}^{\prime}, \ldots, \beta_{n}^{\prime}$ in $V$ are either identical or perpendicular with respect to $\Theta$.

Proof of Proposition 4.2. Let $\mathcal{M}$ be a decomposable Monge-Ampère system with a Lagrangian pair $\left(E_{1}, E_{2}\right)$ on $(M, D)$. First we observe that $\mathcal{M}$ has a decomposable local generator. To see this, let $X_{1}, \ldots, X_{n}$ and $P_{1}, \ldots, P_{n}$ be local frames of $E_{1}$ and $E_{2}$ respectively. Let $R$ be the Reeb vector field for a local contact form $\theta$ defining $D$. Consider the dual coframe $\theta, \alpha_{1}, \ldots, \alpha_{n}, \beta_{1}, \ldots, \beta_{n}$ of $T^{*} M$ to the frame $R, X_{1}, \ldots, X_{n}, P_{1}, \ldots, P_{n}$ of $T M$. Then we see, by the decomposing condition, that there exist an $(n-1)$-form $\gamma$ and a non-vanishing function $\mu$ on $M$ such that $\omega=\mu\left(\beta_{1} \wedge \cdots \wedge \beta_{n}\right)+\theta \wedge \gamma$. Thus we have $\mathcal{M}=\left\langle\beta_{1} \wedge \cdots \wedge \beta_{n}, \theta, d \theta\right\rangle$. Note that $\operatorname{Ann}\left(\theta, \alpha_{1}, \ldots, \alpha_{n}\right)=E_{1}$. Then, by Lemma 4.3 , we see that $\mathcal{M}$ is determined just by $E_{1}$. In particular, given a Lagrangian pair $\left(E_{1}, E_{2}\right)$, the decomposable Monge-Ampère system with the Lagrangian pair $\left(E_{1}, E_{2}\right)$ is uniquely determined.

Remark 4.4. If the characteristic system $E_{1}$ is integrable, then the decomposable MongeAmpère system is isomorphic to the system corresponding to the equation Hess $=0$ (see $[22,24])$. 
Remark 4.5. The equation Hess $=0$ has the infinite-dimensional automorphism pseudogroup which consists of the lifts of diffeomorphisms on the dual projective space. However, if a Lagrangian pair is associated, then the automorphism pseudo-group turns to be of finitedimensional, as stated in Proposition 4.2.

\section{Automorphisms of Monge-Ampère systems with Lagrangian pairs}

Let us consider the automorphism pseudo-group $\operatorname{Aut}(\mathcal{M})$ of all local isomorphisms of a bidecomposable Monge-Ampère system $\mathcal{M}=\langle\theta, d \theta, \omega\rangle$ with a Lagrangian pair $\left(E_{1}, E_{2}\right)$ on a contact manifold $(M, D)$ of dimension $2 n+1$.

By Theorems 3.2 and 3.8, in the case $n \geq 3$, any automorphism of any bi-decomposable Monge-Ampère system with a Lagrangian pair $\left(E_{1}, E_{2}\right)$ preserves the Lagrangian pair $\left(E_{1}, E_{2}\right)$ up to the interchange of $E_{1}, E_{2}$. Therefore infinitesimal symmetries of bi-decomposable MongeAmpère systems are studied based on infinitesimal symmetries of Lagrangian contact structures.

Now let $\bar{G}$ be

$$
\bar{G}=\left\{\left(\begin{array}{ccc}
c^{2} & 0 & 0 \\
b_{1} & c A & \mathrm{O}_{n} \\
b_{2} & \mathrm{O}_{n} & c^{t} A^{-1}
\end{array}\right) \mid c \in \mathbf{R}^{\times}, A \in \mathrm{SL}(n, \mathbf{R}), b_{1}, b_{2} \in \mathbf{R}^{n}\right\} .
$$

Proposition 5.1. The bi-decomposable Monge-Ampère systems with Lagrangian pairs are in bijective correspondence with $\bar{G}$-structures of type $\mathfrak{m}$.

Proof. We consider, as in Section 4, the fundamental GLA of contact type $\mathfrak{m}=\mathfrak{g}_{-2} \oplus \mathfrak{g}_{-1}$ and the decomposition $\mathfrak{g}_{-1}=\mathfrak{e}^{1} \oplus \mathfrak{e}^{2}$ into the Lagrangian pair. Moreover we fix a volume form $\Omega_{1} \in \wedge^{n}\left(\mathfrak{e}^{1 *}\right)$ on $\mathfrak{e}^{1}$ and a volume form $\Omega_{2} \in \wedge^{n}\left(\mathfrak{e}^{2 *}\right)$ on $\mathfrak{e}^{2}$. Consider the group $C\left(\mathfrak{m} ; \mathfrak{e}^{1}, \Omega_{1} ; \mathfrak{e}^{2}, \Omega_{2}\right)$ consisting of all $a \in \mathrm{GL}(\mathfrak{m})$ which satisfies the following conditions: $a \mathfrak{g}_{-1}=\mathfrak{g}_{-1}$, the graded linear automorphism $\bar{a}$ of $\mathfrak{m}$ induced by $a$ is a GLA-automorphism, $a \mathfrak{e}^{1}=\mathfrak{e}^{1}, a \mathfrak{e}^{2}=\mathfrak{e}^{2}$, and $a^{*} \Omega_{1}=\lambda \Omega_{1}, a^{*} \Omega_{2}=\lambda \Omega_{2}$ for some $\lambda \in \mathbf{R}^{\times}$. Then we have that $C\left(\mathfrak{m} ; \mathfrak{e}^{1}, \Omega_{1} ; \mathfrak{e}^{2}, \Omega_{2}\right)$ is identical with $\bar{G}$.

Thus the equivalence problem of bi-decomposable Monge-Ampère systems with Lagrangian pairs is studied as an adapted $G$-structure on a contact manifold of dimension $2 n+1$.

Let $G^{0}$ be a subgroup of $\operatorname{GL}(n+2, \mathbf{R})$ defined by

$$
G^{0}=\left\{\left(\begin{array}{ccc}
k^{-1} & 0 & 0 \\
b_{1} & A & 0 \\
a & { }^{t} b_{2} & k
\end{array}\right) \mid k \in \mathbf{R}^{\times}, A \in \operatorname{SL}(n, \mathbf{R}), b_{1}, b_{2} \in \mathbf{R}^{n}, a \in \mathbf{R}\right\} .
$$

We set

$$
H^{0}=\left\{\left(\begin{array}{ccc}
k^{-1} & 0 & 0 \\
0 & A & 0 \\
0 & 0 & k
\end{array}\right) \mid k \in \mathbf{R}^{\times}, A \in \mathrm{SL}(n, \mathbf{R})\right\} \subset G^{0} .
$$

Then we have the model space $G^{0} / H^{0} \cong \mathbf{R}^{2 n+1}$ with coordinates $(x, z, p)$. The $G^{0}$-action on $\mathbf{R}^{2 n+1}$ is described by

$$
(x, z, p) \mapsto\left(x^{\prime}, z^{\prime}, p^{\prime}\right)=\left(k\left(A x+b_{1}\right), k\left(k z-{ }^{t} b_{2} x-a\right), k\left(p-\frac{1}{k} b_{2}\right) A^{-1}\right) .
$$

Then $d z^{\prime}-p^{\prime} d x^{\prime}=k^{2}(d z-p d x)$ and the form $\omega=c d x_{1} \wedge \cdots \wedge d x_{n}-d p_{1} \wedge \cdots \wedge d p_{n}$ is transformed to $\omega^{\prime}=k^{n} \omega$. 
Let $E_{i, j}, 0 \leq i, j \leq n+1$, denotes the elementary $(n+2) \times(n+2)$-matrix such that the $(i, j)$ component is 1 and other components are all zero. We set

$$
\begin{aligned}
& \varepsilon=-E_{0,0}+E_{n+1, n+1}, \quad e_{i}=E_{i, 0} \quad(1 \leq i \leq n), \\
& f_{j}=E_{n+1, j} \quad(1 \leq j \leq n), \quad \gamma=E_{n+1,0},
\end{aligned}
$$

and identify the set of traceless matrices $\mathfrak{s l}(n, \mathbf{R})$ with

$$
\left\{\left(\begin{array}{ccc}
0 & 0 & 0 \\
0 & A & 0 \\
0 & 0 & 0
\end{array}\right) \mid A \in \mathfrak{s l}(n, \mathbf{R})\right\} \subset \mathfrak{g}^{0} .
$$

Then we set $\mathfrak{g}_{0}=\langle\varepsilon\rangle_{\mathbf{R}} \oplus \mathfrak{s l}(n, \mathbf{R}), \mathfrak{g}_{-1}^{1}=\left\langle e_{1}, \ldots, e_{n}\right\rangle_{\mathbf{R}}, \mathfrak{g}_{-1}^{2}=\left\langle f_{1}, \ldots, f_{n}\right\rangle_{\mathbf{R}}, \mathfrak{g}_{-1}=\mathfrak{g}_{-1}^{1} \oplus \mathfrak{g}_{-1}^{2}$, and $\mathfrak{g}_{-2}=\langle\gamma\rangle_{\mathbf{R}}$. Then

$$
\mathfrak{g}^{0}=\mathfrak{g}_{-2} \oplus \mathfrak{g}_{-1} \oplus \mathfrak{g}_{0}
$$

is the Lie algebra of $G^{0}$. We write $\mathfrak{m}=\mathfrak{g}_{-2} \oplus \mathfrak{g}_{-1}$. Then the Lie subalgebra $\mathfrak{m}$ becomes the split Heisenberg algebra.

The matrix representation of $A_{0} \in \mathfrak{g}_{0}=\langle\varepsilon\rangle_{\mathbf{R}} \oplus \mathfrak{s l}(n, \mathbf{R})$ with respect to the basis $\left\{\gamma, e_{i}\right.$ $\left.(1 \leq i \leq n), f_{j}(1 \leq i \leq n)\right\}$ in the Heisenberg algebra $V=\mathfrak{m}=\mathfrak{g}_{-2} \oplus \mathfrak{g}_{-1}$ has the following form:

$$
A_{0}=C+A_{0}^{\prime}=c\left(\begin{array}{ccc}
2 & 0 & 0 \\
0 & I & \mathrm{O} \\
0 & \mathrm{O} & I
\end{array}\right)+\left(\begin{array}{ccc}
0 & 0 & 0 \\
0 & A & \mathrm{O} \\
0 & \mathrm{O} & -{ }^{t} A
\end{array}\right) .
$$

In fact, for the commutators, we have by the direct calculations:

$$
\begin{array}{ll}
{[\varepsilon, \gamma]=2 \gamma,} & {\left[\varepsilon, e_{i}\right]=e_{i}, \quad\left[\varepsilon, f_{j}\right]=f_{j},} \\
{[A, \gamma]=O,} & {\left[A, e_{i}\right]=a_{i}, \quad\left[A, f_{j}\right]=-a^{j},}
\end{array}
$$

where $a_{i}$ is the $i$-th column of $A$ and $a^{j}$ is the $j$-th row of $A, 1 \leq i, j \leq n$, and $A \in \mathfrak{s l}(n, \mathbf{R})$. Moreover we have

$$
\left[e_{i}, f_{j}\right]=-\delta_{i j} \gamma, \quad\left[e_{i}, e_{j}\right]=0, \quad\left[f_{i}, f_{j}\right]=0 \quad(1 \leq i, j \leq n) .
$$

We will study the prolongation of $\left(\mathfrak{m}, \mathfrak{g}_{0}\right)$. We define the prolongation inductively $\mathfrak{g}_{k}=$ $\mathfrak{g}\left(\mathfrak{m}, \mathfrak{g}_{0}\right)_{k}(k \geq 1)$ by the set of elements $\left\{(\alpha, \beta) \in \operatorname{Hom}\left(\mathfrak{g}_{-1}, \mathfrak{g}_{k-1}\right) \oplus \operatorname{Hom}\left(\mathfrak{g}_{-2}, \mathfrak{g}_{k-2}\right)\right\}$ satisfying

(i) $\beta([x, y])=[\alpha(x), y]-[\alpha(y), x] \quad\left(x, y \in \mathfrak{g}_{-1}\right)$,

(ii) $[\alpha(y), z]=[\beta(z), y] \quad\left(y \in \mathfrak{g}_{-1}, z \in \mathfrak{g}_{-2}\right)$.

See [31, p. 429]. Then we have

Lemma 5.2. The prolongation $\mathfrak{g}_{i}$ vanishes for any $i \geq 1$.

Proof. First we calculate $\mathfrak{g}_{1}$. For any $(\alpha, \beta) \in \operatorname{Hom}\left(\mathfrak{g}_{-1}, \mathfrak{g}_{0}\right) \oplus \operatorname{Hom}\left(\mathfrak{g}_{-2}, \mathfrak{g}_{-1}\right)$, the conditions (i) and (ii) imply that

(i) $\beta\left(\left[e_{i}, f_{j}\right]\right)=\left[\alpha\left(e_{i}\right), f_{j}\right]-\left[\alpha\left(f_{j}\right), e_{i}\right] \quad(1 \leq i, j \leq n)$,

(ii-1) $\left[\alpha\left(e_{i}\right), \gamma\right]=\left[\beta(\gamma), e_{i}\right] \quad(1 \leq i \leq n)$,

(ii-2) $\left[\alpha\left(f_{j}\right), \gamma\right]=\left[\beta(\gamma), f_{j}\right] \quad(1 \leq j \leq n)$. 
Set

$$
\beta(\gamma)=\sum_{\ell=1}^{n} b_{\ell} e_{\ell}+\sum_{m=1}^{n} c_{m} f_{m}, \quad \alpha\left(e_{i}\right)=h_{i} \varepsilon+A^{(i)}, \quad \alpha\left(f_{j}\right)=k_{j} \varepsilon+B^{(j)},
$$

where $b_{\ell}, c_{m}, h_{i}, k_{j} \in \mathbf{R}, A^{(i)}, B^{(j)} \in \mathfrak{s l}(n, \mathbf{R})$. Then

$$
\begin{aligned}
& \beta\left(\left[e_{i}, f_{j}\right]\right)=\sum_{\ell=1}^{n}\left(-\delta_{i j} b_{\ell}\right) e_{\ell}+\sum_{m=1}^{n}\left(-\delta_{i j} c_{m}\right) f_{m}, \\
& {\left[\alpha\left(e_{i}\right), f_{j}\right]=h_{i} f_{j}-\left(j \text {-th row of } A^{(i)}\right), \quad\left[\alpha\left(f_{j}\right), e_{i}\right]=k_{j} e_{i}+\left(i \text {-th column of } B^{(j)}\right),} \\
& {\left[\alpha\left(e_{i}\right), \gamma\right]=2 h_{i} \gamma, \quad\left[\beta(\gamma), e_{i}\right]=c_{i} \gamma, \quad\left[\alpha\left(f_{j}\right), \gamma\right]=2 k_{j} \gamma, \quad\left[\beta(\gamma), f_{j}\right]=-b_{j} \gamma .}
\end{aligned}
$$

By the condition (i), we have

$$
B_{\ell i}^{(j)}=-\delta_{\ell i} k_{j}+\delta_{i j} b_{\ell}, \quad A_{j m}^{(i)}=\delta_{j m} h_{i}+\delta_{i j} c_{m} \quad(1 \leq i, j, \ell, m \leq n) .
$$

By the condition (ii-1), we have $c_{i}=2 h_{i}, 1 \leq i \leq n$. By the condition (ii-2), we have $b_{j}=-2 k_{j}$, $1 \leq j \leq n$. Therefore we have

$$
B_{\ell i}^{(j)}=-\delta_{\ell i} k_{j}-2 \delta_{i j} k_{\ell}, \quad A_{j m}^{(i)}=\delta_{j m} h_{i}+2 \delta_{i j} h_{m} \quad(1 \leq i, j, \ell, m \leq n) .
$$

In particular, for any $j, \ell$ with $1 \leq j, \ell \leq n$, we have $B_{\ell \ell}^{(j)}=-k_{j}-2 \delta_{\ell j} k_{\ell}$, and therefore

$$
0=\operatorname{tr} B^{(j)}=-(n+2) k_{j}
$$

hence $k_{j}=0,1 \leq j \leq n$. For any $i, m$ with $1 \leq i, m \leq n$, we have $A_{m m}^{(j)}=h_{i}+2 \delta_{i m} h_{m}$, and therefore

$$
0=\operatorname{tr} A^{(i)}=(n+2) h_{i},
$$

hence $h_{i}=0,1 \leq i \leq n$. Thus we have $\beta=0$ and $\alpha=0$. Therefore we have $\mathfrak{g}_{1}=0$.

Second we calculate $\mathfrak{g}_{2}$. Take any $(\alpha, \beta) \in \mathfrak{g}_{2} \subset \operatorname{Hom}\left(\mathfrak{g}_{-1}, \mathfrak{g}_{1}\right) \oplus \operatorname{Hom}\left(\mathfrak{g}_{-2}, \mathfrak{g}_{0}\right)$. Since $\mathfrak{g}_{1}=0$, $\alpha=0$. Then we see $\beta(\gamma)=0$. Therefore $\beta=0$. Thus we obtain that $\mathfrak{g}_{2}=0$.

From $\mathfrak{g}_{1}=0, \mathfrak{g}_{2}=0$, we have $\mathfrak{g}_{i}=0, i \geq 3$, automatically.

Then we have

Theorem 5.3. Let $(M, D)$ be a contact manifold of dimension $2 n+1$ and $\mathcal{M}$ a bi-decomposable Monge-Ampère system with a Lagrangian pair on $(M, D)$. Assume that $n \geq 3$. Then the automorphism pseudo-group $\operatorname{Aut}(\mathcal{M})$ of $\mathcal{M}$ has at most the dimension of $(n+1)^{2}$. The estimate is best possible.

Proof. By Lemma 5.2, we have known that the prolongations $\mathfrak{g}_{i}, 1 \leq i$ in the sense of Tanaka vanish. On the other hand $\bar{G}$ is equal to $\left(H^{0}\right)^{\#}$ in the notation of [29]. Then, by the finiteness theorem of Tanaka [29, Corollary 2], we see that the dimension of the pseudo-group of automorphisms on $\mathcal{M}$ is estimated by $\operatorname{dim}\left(\mathfrak{g}^{0}\right)=\sum_{i \leq 0} \operatorname{dim}\left(\mathfrak{g}_{i}\right)=(n+1)^{2}$. Moreover there exists an Monge-Ampère system $\mathcal{M}$ with a Lagrangian pair on $M=\mathbf{R}^{2 n+1}$, which arises in equi-affine geometry, such that the automorphism group $\operatorname{Aut}(\mathcal{M})$ attains the maximal dimension $(n+1)^{2}$. See Section 8.1.

Remark 5.4. The sharp symmetry bounds of non-flat Lagrangian contact structures together with many other parabolic geometries have been obtained by Kruglikov and The [18]. Lemma 5.2 in our paper is similar to the concept of prolongation rigidity studied by them in [18]. 


\section{Hesse representations}

Let $\left(E_{1}, E_{2}\right)$ be a Lagrangian pair on a contact manifold $(M, D)$. We call $\left(E_{1}, E_{2}\right)$ bi-Legendreintegrable or simply integrable if both $E_{1}$ and $E_{2}$ are completely integrable as subbundles in $T M$. Then $\left(E_{1}, E_{2}\right)$ defines a pair of Legendrian foliations $\left(\mathcal{E}_{1}, \mathcal{E}_{2}\right)$ on $M$ locally. The standard Lagrangian pair $\left(E_{1}^{\mathrm{st}}, E_{2}^{\mathrm{st}}\right)$ introduced in Section 2 is integrable. In fact the foliation $\mathcal{E}_{1}^{\text {st }}$ is defined by fibers of the projection $(x, z, p) \mapsto\left(p, \sum_{i=1}^{n} x_{i} p_{i}-z\right)$ and the foliation $\mathcal{E}_{2}^{\text {st }}$ is defined by $(x, z, p) \mapsto(x, z)$.

Definition 6.1. If a Lagrangian pair $\left(E_{1}, E_{2}\right)$ on $(M, D)$ is locally contactomorphic to the standard Lagrangian pair $\left(E_{1}^{\mathrm{st}}, E_{2}^{\mathrm{st}}\right)$ on $\left(\mathbf{R}^{2 n+1}, D_{\mathrm{st}}\right)$, then we call $\left(E_{1}, E_{2}\right)$ flat.

Let $\left(E_{1}, E_{2}\right)$ be a Lagrangian pair on a contact manifold $(M, D)$ of dimension $2 n+1$. Assume that $\left(E_{1}, E_{2}\right)$ is bi-Legendre-integrable. Then, locally, there exist Legendrian fibrations $\pi_{1}: M \rightarrow W_{1}$ and $\pi_{2}: M \rightarrow W_{2}$ having $E_{2}$ and $E_{1}$ as the kernels of the differentials $\left(\pi_{1}\right)_{*}: T M \rightarrow$ $T W_{1}$ and $\left(\pi_{2}\right)_{*}: T M \rightarrow T W_{2}$ for some manifolds $W_{1}$ and $W_{2}$ of dimension $n+1$ respectively. Then we have the following diagram:

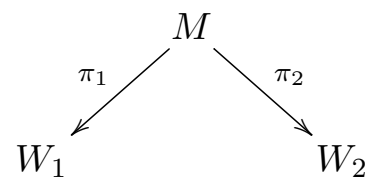

We call $\left(\pi_{1}, \pi_{2}\right)$ a double Legendrian fibration. In this case we say that $W_{1}$ and $W_{2}$ are in the dual relation via the Legendre transformation on $M$. Since $D=E_{1} \oplus E_{2}$, we see $\left(\pi_{1}, \pi_{2}\right): M \rightarrow$ $W_{1} \times W_{2}$ is an immersion. Thus $M$ has a pseudo-product structure doubly foliated by Legendrian submanifolds $[27,30]$.

Example 6.2. The projective cotangent bundle $M=P\left(T^{*} W\right)$ over a manifold $W$ of dimension $n+1$ with the canonical projection $\pi_{1}: M \rightarrow W$ has the canonical contact structure $D \subset T M$ defined by, for any $(x,[\alpha]) \in M$ with $x \in W,[\alpha] \in P\left(T_{x}^{*} W\right)$,

$$
D_{(x,[\alpha])}=\left\{v \in T_{x} M \mid \alpha\left(\left(\pi_{1}\right)_{*} v\right)=0\right\} .
$$

We see moreover that $D$ has the integrable Lagrangian subbundle $E_{2}=\operatorname{Ker}\left(\pi_{1 *}\right)$.

Assume that $W$ is the projective space $P^{n+1}$. Then the contact manifold $M=P\left(T^{*} P^{n+1}\right)$ is naturally identified with the contact manifold $P\left(T^{*} P^{n+1^{*}}\right)$, the projective cotangent bundle over the dual projective space $P^{n+1^{*}}$ with the canonical projection $\pi_{2}: P\left(T^{*} P^{n+1^{*}}\right) \rightarrow P^{n+1^{*}}[13]$. Then $D \subset T M$ has another Lagrangian subbundle $E_{1}=\operatorname{Ker}\left(\left(\pi_{2}\right)_{*}\right)$ and $\left(E_{1}, E_{2}\right)$ turns to be an integrable Lagrangian pair of $(M, D)$. There is associated to $\left(E_{1}, E_{2}\right)$ the double Legendrian fibration

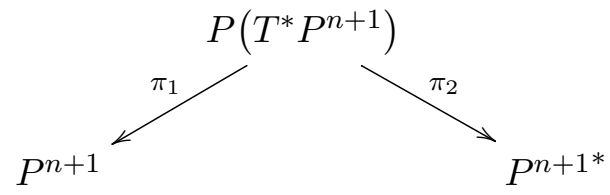

for $W_{1}=P^{n+1}, W_{2}=P^{n+1^{*}}$. This globally defined Lagrangian pair $\left(E_{1}, E_{2}\right)$ is flat. In fact, $P\left(T^{*} P^{n+1}\right)$ is identified with the incidence hypersurface $I \subset P^{n+1} \times P^{n+1^{*}}$ defined by

$$
x_{0} y_{0}+x_{1} y_{1}+\cdots+x_{n+1} y_{n+1}=0
$$


for homogeneous coordinates $[x]=\left[x_{0}: x_{1}: \cdots: x_{n+1}\right]$ of $P^{n+1}$ and $[y]=\left[y_{0}: y_{1}: \cdots: y_{n+1}\right]$ of $P^{n+1^{*}}$. On the affine open subset $U=\left\{x_{0} \neq 0, y_{n+1} \neq 0\right\} \subset P^{n+1} \times P^{n+1^{*}}$, take local coordinates of

$$
x_{i}^{\prime}=\frac{x_{i}}{x_{0}}, \quad z^{\prime}=-\frac{x_{n+1}}{x_{0}}, \quad p_{j}^{\prime}=\frac{y_{j}}{y_{n+1}}, \quad \tilde{z}^{\prime}=-\frac{y_{0}}{y_{n+1}},
$$

$(1 \leq i \leq n, 1 \leq j \leq n)$. Then $I \cap U$ is defined by $-z^{\prime}-\tilde{z}^{\prime}+\sum_{i=1}^{n} x_{i}^{\prime} p_{i}^{\prime}=0$, where we have $d z^{\prime}-\sum_{i=1}^{n} p_{i}^{\prime} d x_{i}^{\prime}+\left(d \tilde{z}^{\prime}-\sum_{i=1}^{n} x_{i}^{\prime} d p_{i}^{\prime}\right)=0$. The contact structure on $I \cap U$ is given by $d z^{\prime}-\sum_{i=1}^{n} p_{i}^{\prime} d x_{i}^{\prime}=0$. Also it is given by $d \tilde{z}^{\prime}-\sum_{i=1}^{n} x_{i}^{\prime} d p_{i}^{\prime}=0$. This shows $\left.\left(E_{1}, E_{2}\right)\right|_{U}$ is flat. Also on other affine open subsets, we can verify the flatness of $\left(E_{1}, E_{2}\right)$ similarly or by an argument using homogeneity.

Example 6.3. For a Riemannian manifold $W=(W, g)$ of dimension $n+1$, the unit tangent bundle $M=T_{1} W$ of $W$ has the canonical contact structure $D \subset T M$,

$$
D_{(x, v)}=\left\{u \in T_{(x, v)} M \mid \pi_{*} u \in v^{\perp}\right\}, \quad(x, v) \in T_{1} W,
$$

and the canonical Lagrangian pair $\left(E_{1}, E_{2}\right)$ induced by the horizontal lift and the vertical lift of the Levi-Civita connection:

$$
\left(E_{1}\right)_{(x, v)}=\left(v^{\perp}\right)^{\text {hor }}, \quad\left(E_{2}\right)_{(x, v)}=\left(v^{\perp}\right)^{\text {ver }}, \quad(x, v) \in T_{1} W .
$$

Here $\pi: M \longrightarrow W$ is the canonical projection and $v^{\perp}=\left\{w \in T_{x} W \mid g(v, w)=0\right\}$. Then $\left(E_{1}, E_{2}\right)$ is bi-Legendre-integrable if and only if $W$ is a space form. In fact, the vertical lift $E_{2}$ is always completely integrable. Moreover the horizontal lift $E_{1}$ is completely integrable if and only if $W$ is projectively flat, that is, a space form (see [28, Corollaries 3.5 and 6.5]).

The bi-decomposable class of Monge-Ampère systems with flat Lagrangian pairs turns out to be an intrinsic representation of the well-known class of Monge-Ampère equations locally expressed by Hesse representations:

$$
\operatorname{Hess}(z)=F\left(x_{1}, \ldots, x_{n}, z, p_{1}, \ldots, p_{n}\right) \quad(\neq 0) .
$$

Thus we are led to the following definition:

Definition 6.4. We call a Monge-Ampère system with a flat Lagrangian pair a Hesse MongeAmpère system.

Note that there are sub-classes of Hesse Monge-Ampère equations, Euler-Lagrange MongeAmpère equations:

$$
\operatorname{Hess}(z)=F_{1}\left(x_{1}, \ldots, x_{n}, z\right) \cdot F_{2}\left(p_{1}, \ldots, p_{n}, \sum_{j=1}^{n} p_{j} x_{j}-z\right) \quad(\neq 0),
$$

(see [5, p. 21, Example 2]) and flat Monge-Ampère equations:

$$
\operatorname{Hess}(z)=c \quad(c \text { is constant, } c \neq 0) .
$$

Definition 6.5. Let $\mathcal{M}$ be a Hesse Monge-Ampère system. Then we call $\mathcal{M}$ an Euler-Lagrange Monge-Ampère system if $\mathcal{M}$ is locally generated by a bi-decomposable $n$-form $\omega=\omega_{1}-\omega_{2}$ satisfying

$$
d \omega_{1} \equiv 0, \quad d \omega_{2} \equiv 0 \quad \bmod \theta
$$


Thus we have a sequence of classes of Monge-Ampère systems:

$\{$ M-A system with Lagrangian pair $\} \supset\{$ M-A system with integrable Lag. pair $\}$

$\supset\{$ Hesse M-A system $\} \supset$ \{Euler-Lagrange M-A system $\} \supset$ \{flat M-A system\}.

We will study Hesse Monge-Ampère systems in detail, and, in fact, we characterize the above three classes in intrinsic way. Note that the equation of non-zero constant Gauss-Kronecker curvature $K=c$ falls into the class of Euler-Lagrange Monge-Ampère systems and the equation of improper affine hyperspheres Hess $=c$ falls into the class of flat Monge-Ampère systems.

We will show, in Sections 8.2-8.5, that Monge-Ampère systems defined by the Gaussian curvature constant equation $K=c$ in $\mathbf{E}^{n+1}, S^{n+1}, H^{n+1}$ are Euler-Lagrange systems.

Proposition 6.6. Let $\mathcal{M}$ be a Hesse Monge-Ampère system, i.e., a Monge-Ampère system with a flat Lagrangian pair $\left(E_{1}, E_{2}\right)$ generated by an $n$-form $\omega=\omega_{1}-\omega_{2}$ enjoying the bi-decomposing condition. Then $\mathcal{M}$ is locally isomorphic to a Monge-Ampère system $\mathcal{M}^{\prime}$ on an open subset $U \subset \mathbf{R}^{2 n+1}$ with the standard Lagrangian pair $\left(E_{1}^{\text {st }}, E_{2}^{\text {st }}\right)$ which is locally generated by an $n$-form of type

$$
F\left(x_{1}, \ldots, x_{n}, z, p_{1}, \ldots, p_{n}\right) d x_{1} \wedge \cdots \wedge d x_{n}-d p_{1} \wedge \cdots \wedge d p_{n}
$$

for a non-vanishing function $F$ on $U$. In particular, there exists a system of local Darboux coordinates $\left(x_{1}, \ldots, x_{n}, z, p_{1}, \ldots, p_{n}\right)$ in some neighborhood of each point, such that $\mathcal{M}$ is represented by a Hesse Monge-Ampère equation of the form

$$
\operatorname{Hess}(z)=F\left(x_{1}, \ldots, x_{n}, z, p_{1}, \ldots, p_{n}\right) \quad(F \neq 0) \text {. }
$$

Proof. Since $\left(E_{1}, E_{2}\right)$ is flat, around each point of $M$, there exists a system of local coordinates $\left(x_{1}, \ldots, x_{n}, z, p_{1}, \ldots, p_{n}\right)$ such that $D=\{\theta=0\}, \theta=d z-\sum_{i=1}^{n} p_{i} d x_{i}$, and that

$$
\begin{aligned}
& E_{1}=\operatorname{Ker}\left(\pi_{2 *}\right)=\left\langle\frac{\partial}{\partial x_{1}}+p_{1} \frac{\partial}{\partial z}, \ldots, \frac{\partial}{\partial x_{n}}+p_{n} \frac{\partial}{\partial z}\right\rangle, \\
& E_{2}=\operatorname{Ker}\left(\pi_{1 *}\right)=\left\langle\frac{\partial}{\partial p_{1}}, \ldots, \frac{\partial}{\partial p_{n}}\right\rangle, \\
& \pi_{1}: \mathbf{R}^{2 n+1} \rightarrow \mathbf{R}^{n+1}, \quad \pi_{1}\left(x_{1}, \ldots, x_{n}, z, p_{1}, \ldots, p_{n}\right)=\left(x_{1}, \ldots, x_{n}, z\right), \\
& \pi_{2}: \mathbf{R}^{2 n+1} \rightarrow \mathbf{R}^{n+1}, \quad \pi_{2}\left(x_{1}, \ldots, x_{n}, z, p_{1}, \ldots, p_{n}\right)=\left(p_{1}, \ldots, p_{n}, \sum_{i=1}^{n} p_{i} x_{i}-z\right) .
\end{aligned}
$$

This means that $\mathcal{M}$ is locally isomorphic to a Monge-Ampère system with the standard Lagrangian pair. Then, from the bi-decomposing condition, we have, setting $x=\left(x_{1}, \ldots, x_{n}\right)$, $p=\left(p_{1}, \ldots, p_{n}\right)$,

$$
\omega=\omega_{1}-\omega_{2}=f(x, z, p) d x_{1} \wedge \cdots \wedge d x_{n}-g(x, z, p) d p_{1} \wedge \cdots \wedge d p_{n}
$$

for some functions $f(x, z, p), g(x, z, p)(\neq 0)$ on $\mathbf{R}^{2 n+1}$. Therefore, putting $F=f / g$, we have a form $\operatorname{Hess}(z)=F(x, z, p)$.

Proposition 6.7. Let $\mathcal{M}$ be a Hesse Monge-Ampère system. Then $\mathcal{M}$ is an Euler-Lagrange Monge-Ampère system if and only if $\mathcal{M}$ is locally isomorphic to a Monge-Ampère system $\mathcal{M}^{\prime}$ on an open subset $U \subset \mathbf{R}^{2 n+1}$ with the standard Lagrangian pair generated by an $n$-form of type

$$
F_{1}\left(x_{1}, \ldots, x_{n}, z\right) \cdot F_{2}\left(\widetilde{z}, p_{1}, \ldots, p_{n}\right) d x_{1} \wedge \cdots \wedge d x_{n}-d p_{1} \wedge \cdots \wedge d p_{n}
$$


for some non-vanishing functions $F_{1}$ of $x, z$ and $F_{2}$ of $\widetilde{z}=\sum_{j=1}^{n} p_{j} x_{j}-z$ and $p$. In particular,

$\mathcal{M}$ is locally represented as

$$
\operatorname{Hess}(z)=F_{1}\left(x_{1}, \ldots, x_{n}, z\right) \cdot F_{2}\left(\widetilde{z}, p_{1}, \ldots, p_{n}\right) \quad\left(F_{1}, F_{2} \neq 0\right) \text {. }
$$

Proof. By the assumption, we can set

$$
\omega_{1}=f(x, z, p) d x_{1} \wedge \cdots \wedge d x_{n}, \quad \omega_{2}=g(x, z, p) d p_{1} \wedge \cdots \wedge d p_{n} .
$$

Since

$$
\begin{aligned}
d \omega_{1} & =d f \wedge d x_{1} \wedge \cdots \wedge d x_{n}=\left(\sum_{i=1}^{n} \frac{\partial f}{\partial x_{i}} d x_{i}+\frac{\partial f}{\partial z} d z+\sum_{i=1}^{n} \frac{\partial f}{\partial p_{i}} d p_{i}\right) \wedge d x_{1} \wedge \cdots \wedge d x_{n} \\
& \equiv \sum_{i=1}^{n} \frac{\partial f}{\partial p_{i}} d p_{i} \wedge d x_{1} \wedge \cdots \wedge d x_{n}, \quad \bmod \theta
\end{aligned}
$$

we see $d \omega_{1} \equiv 0 \bmod \theta$ if and only if $f$ is independent of $p_{1}, \ldots, p_{n}: f=f\left(x_{1}, \ldots, x_{n}, z\right)$. Besides, we take another system of local coordinates $(x, \tilde{z}, p)$ with $\tilde{z}=\sum_{i=1}^{n} x_{i} p_{i}-z$. Then $\theta=-\left(d \tilde{z}-\sum_{i=1}^{n} x_{i} d p_{i}\right)$ and we have

$$
\begin{aligned}
d \omega_{2} & =d g \wedge d p_{1} \wedge \cdots \wedge d p_{n}=\left(\sum_{i=1}^{n} \frac{\partial g}{\partial x_{i}} d x_{i}+\frac{\partial g}{\partial \tilde{z}} d \tilde{z}+\sum_{i=1}^{n} \frac{\partial g}{\partial p_{i}} d p_{i}\right) \wedge d p_{1} \wedge \cdots \wedge d p_{n} \\
& \equiv \sum_{i=1}^{n} \frac{\partial g}{\partial x_{i}} d x_{i} \wedge d p_{1} \wedge \cdots \wedge d p_{n}, \quad \bmod \theta
\end{aligned}
$$

Therefore we see $d \omega_{2} \equiv 0 \bmod \theta$ if and only if $g$ is independent of $x$ for the system of local coordinates $(x, \tilde{z}, p): g=g\left(\widetilde{z}, p_{1}, \ldots, p_{n}\right)$. Therefore putting $F_{1}=f, F_{2}=1 / g$, we have a form $\operatorname{Hess}(z)=F_{1}(x, z) \cdot F_{2}(\widetilde{z}, p)$.

By Theorem 3.8, we have the following:

Proposition 6.8. Let $n \geq 3$. Then Definition 6.4 (resp. Definition 6.5) depends only on the Monge-Ampère system and does not depend on the choice of Lagrangian pairs of the MongeAmpère system. The class of Hesse Monge-Ampère systems (resp. the class of Euler-Lagrange Monge-Ampère systems) is invariant under contact transformations.

Proof. Let $\mathcal{M}$ be a Monge-Ampère system with a Lagrangian pair on a contact manifold $(M, D)$. Let $\left(E_{1}, E_{2}\right)$ and $\left(E_{1}^{\prime}, E_{2}^{\prime}\right)$ be two Lagrangian pairs associated to $\mathcal{M}$. Then, by Theorem 3.8, $E_{1}^{\prime}=E_{1}, E_{2}^{\prime}=E_{2}$ or $E_{1}^{\prime}=E_{2}, E_{2}^{\prime}=E_{1}$. Therefore the flatness of Lagrangian pair depends only on $\mathcal{M}$. Moreover it is clear that, the condition of Definition 6.5, i.e., the possibility of a bi-decomposition $\omega=\omega_{1}-\omega_{2}$ of a local generator $\omega$ into closed decomposable forms $\omega_{1}, \omega_{2}$ up to a contact form $\theta$ depends only on $\mathcal{M}$.

Let $\Phi:(M, D) \rightarrow\left(M^{\prime}, D^{\prime}\right)$ be a contact transformation between $(M, D)$ and another contact manifold $\left(M^{\prime}, D^{\prime}\right)$. Set $\mathcal{M}^{\prime}=\Phi^{-1^{*}} \mathcal{M}$. Then $\mathcal{M}^{\prime}$ is a Monge-Ampère system with the Lagrangian pair $\left(\Phi_{*} E_{1}, \Phi_{*} E_{2}\right)$. If $\left(E_{1}, E_{2}\right)$ is flat, then so is $\left(\Phi_{*} E_{1}, \Phi_{*} E_{2}\right)$. Moreover if $\omega=\omega_{1}-\omega_{2}$ is a bi-decomposition satisfying the condition of Definition 6.5 for $\mathcal{M}$, then $\left(\Phi^{-1}\right)^{*} \omega=\left(\Phi^{-1}\right)^{*} \omega_{1}-\left(\Phi^{-1}\right)^{*} \omega_{2}$ satisfies the condition of Definition 6.5 for $\mathcal{M}^{\prime}$. 
Remark 6.9. The Euler-Lagrange systems are studied in [5] via the key notion "PoincaréCartan form". If an Euler-Lagrange Monge-Ampère system with Lagrangian pair is given by

$$
\begin{aligned}
& \theta=d z-\sum_{i=1}^{n} p_{i} d x_{i}=-\left(d \tilde{z}-\sum_{i=1}^{n} x_{i} d p_{i}\right) \\
& \omega=f(x, z) d x_{1} \wedge \cdots \wedge d x_{n}-g(p, \tilde{z}) d p_{1} \wedge \cdots \wedge d p_{n}
\end{aligned}
$$

then the Poincaré-Cartan form is given by

$$
\Pi=\theta \wedge \omega=f(x, z) d z \wedge d x_{1} \wedge \cdots \wedge d x_{n}-g(p, \tilde{z}) d \tilde{z} \wedge d p_{1} \wedge \cdots \wedge d p_{n} .
$$

To conclude this section, we characterize, among others, the class of equations Hess $=c$ in term of the projective structure:

Proposition 6.10. Let $\mathcal{M}$ be an Euler-Lagrange Monge-Ampère system on $M=P\left(T^{*} P^{n+1}\right)$ induced by the diagram

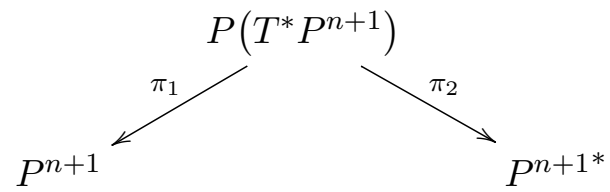

and $W_{1}=P^{n+1}, W_{2}=P^{n+1^{*}}$, generated by a bi-decomposable $n$-form $\omega=\omega_{1}-\omega_{2}$. Then the condition

$$
\nabla \omega_{1}=0, \quad \nabla \omega_{2}=0
$$

is satisfied for the covariant derivative $\nabla$ of the flat connection induced on each local projective chart of $W_{1}=P^{n+1}$ if and only if $\mathcal{M}$ is represented by a Monge-Ampère equation

$$
\operatorname{Hess}(z)=c \quad(c \text { is constant, } c \neq 0) .
$$

Proof. From the equivalence between the conditions $\nabla \omega_{1}=0$ and $d f=0$ (resp. $\nabla \omega_{2}=0$ and $d g=0)$ in the proof of Proposition 6.6, we have that $f, g$ are non-zero constants. Therefore we have the form $\operatorname{Hess}(z)=c(\neq 0)$.

\section{$7 \quad$ A method to construct Monge-Ampère systems with Lagrangian pairs}

Let $(M, D)$ be a contact manifold of dimension $2 n+1$ and $\left(E_{1}, E_{2}\right)$ a Lagrangian pair on $(M, D)$. Consider the quotient bundle $T M / E_{2}$ (resp. $\left.T M / E_{1}\right)$ of rank $n+1$ and a section $\omega_{1}^{\prime}$ (resp. $\left.\omega_{2}^{\prime}\right)$ to the line bundle $\wedge^{n+1}\left(T M / E_{2}\right)^{*}$ (resp. $\left.\wedge^{n+1}\left(T M / E_{1}\right)^{*}\right)$ off the zero-section. Let $\theta$ be a local contact form defining $D$. Recall that the Reeb vector field $R=R_{\theta}$ is defined by the condition $i_{R_{\theta}} \theta=1, i_{R_{\theta}} d \theta=0$. Then we define an $n$-form on $M$ by

$$
\omega=i_{R_{\theta}}\left(\Pi_{1}^{*} \omega_{1}^{\prime}-\Pi_{2}^{*} \omega_{2}^{\prime}\right)
$$

Here $\Pi_{1}: T M \rightarrow T M / E_{2}$ (resp. $\Pi_{2}: T M \rightarrow T M / E_{1}$ ) denotes the bundle projection, and $\Pi_{1}^{*}:\left(T M / E_{2}\right)^{*} \rightarrow T^{*} M$ and $\Pi_{1}^{*}: \wedge^{n+1}\left(T M / E_{2}\right)^{*} \rightarrow \wedge^{n+1} T^{*} M\left(\right.$ resp. $\Pi_{2}^{*}:\left(T M / E_{1}\right)^{*} \rightarrow T^{*} M$ and $\left.\Pi_{2}^{*}: \wedge^{n+1}\left(T M / E_{1}\right)^{*} \rightarrow \wedge^{n+1} T^{*} M\right)$ its dual injections. Then we have the following basic lemma for our construction: 
Lemma 7.1. The differential system $\mathcal{M}=\langle\theta, d \theta, \omega\rangle$, generated by $\omega$ and the contact form $\theta$, is independent of the choice of $\theta$, and depends only on given $\omega_{1}^{\prime}, \omega_{2}^{\prime}$.

We call $\mathcal{M}$ the Monge-Ampère system with $\left(E_{1}, E_{2}\right)$ induced from $\omega_{1}^{\prime}, \omega_{2}^{\prime}$.

To show Lemma 7.1, take a local symplectic frame $X_{1}, \ldots, X_{n}, P_{1}, \ldots, P_{n}$ of $D$ with respect to $d \theta$ :

$$
d \theta\left(X_{i}, X_{j}\right)=0, \quad d \theta\left(P_{i}, P_{j}\right)=0, \quad d \theta\left(X_{i}, P_{j}\right)=\delta_{i j},
$$

with

$$
E_{1}=\left\langle X_{1}, \ldots, X_{n}\right\rangle, \quad E_{2}=\left\langle P_{1}, \ldots, P_{n}\right\rangle
$$

Then $X_{1}, \ldots, X_{n}, P_{1}, \ldots, P_{n}, R_{\theta}$ form a local frame of $T M$.

Lemma 7.2. The Reeb vector field $R_{\theta^{\prime}}$ for a contact form $\theta^{\prime}=\rho \theta$ defining $D$ is given by

$$
R_{\theta^{\prime}}=\frac{1}{\rho^{2}}\left[\sum_{i=1}^{n}\left(P_{i} \rho\right) X_{i}-\sum_{i=1}^{n}\left(X_{i} \rho\right) P_{i}+\rho R_{\theta}\right]
$$

Proof. Let $\alpha_{1}, \ldots, \alpha_{n}, \beta_{1}, \ldots, \beta_{n}, \theta$ be the frame of $T^{*} M$ dual to $X_{1}, \ldots, X_{n}, P_{1}, \ldots, P_{n}, R_{\theta}$. Set $R_{\theta^{\prime}}=\sum_{i} a_{i} X_{i}+\sum_{j} b_{j} P_{j}+c R_{\theta}$. Then, by $i_{R_{\theta^{\prime}}} \theta^{\prime}=1$, we have $c=\frac{1}{\rho}$. Besides we have

$$
\begin{aligned}
i_{R_{\theta^{\prime}}} d \theta^{\prime} & =\left[i_{R_{\theta^{\prime}}} d \rho\right] \theta-i_{R_{\theta^{\prime}}} \theta d \rho+\rho i_{R_{\theta^{\prime}}} d \theta \\
& =\left[\sum_{i} a_{i}\left(X_{i} \rho\right)+\sum_{j} b_{j}\left(P_{j} \rho\right)+c\left(R_{\theta} \rho\right)\right] \theta-c d \rho+\rho\left[\sum_{i} a_{i} \beta_{i}-\sum_{j} b_{j} \alpha_{j}\right],
\end{aligned}
$$

while $d \rho=\sum_{i}\left(X_{i} \rho\right) \alpha_{i}+\sum_{j}\left(P_{j} \rho\right) \beta_{j}+\left(R_{\theta} \rho\right) \theta$. Therefore, by $i_{R_{\theta^{\prime}}} d \theta^{\prime}=0$, we have

$$
-\sum_{i}\left[\rho b_{i}+c\left(X_{i} \rho\right)\right] \alpha_{i}+\sum_{j}\left[\rho a_{j}-c\left(P_{j} \rho\right)\right] \beta_{j}+\left[\sum_{i} a_{i}\left(X_{i} \rho\right)+\sum_{j} b_{j}\left(P_{j} \rho\right)\right] \theta=0 .
$$

Thus we have

$$
a_{i}=\frac{1}{\rho^{2}} P_{i} \rho, \quad b_{i}=-\frac{1}{\rho^{2}} X_{i} \rho, \quad c=\frac{1}{\rho} .
$$

Proof of Lemma 7.1. We set $\Pi_{1}^{*} \omega^{\prime}{ }_{1}=\lambda \cdot \theta \wedge \alpha_{1} \wedge \cdots \wedge \alpha_{n}$ for a function $\lambda$. Then $i_{R_{\theta}}\left(\Pi_{1}^{*} \omega^{\prime}{ }_{1}\right)=$ $\lambda \cdot \alpha_{1} \wedge \cdots \wedge \alpha_{n}$. By Lemma 7.2, we have for $\theta^{\prime}$

$$
i_{R_{\theta^{\prime}}}\left(\Pi_{1}^{*} \omega^{\prime}\right) \equiv \frac{1}{\rho} \lambda \cdot \alpha_{1} \wedge \cdots \wedge \alpha_{n}=\frac{1}{\rho} \cdot i_{R_{\theta}} \omega_{1}^{\prime}, \quad \bmod \theta .
$$

Similarly we have $i_{R_{\theta^{\prime}}}\left(\Pi_{2}^{*} \omega_{2}^{\prime}\right) \equiv \frac{1}{\rho} \cdot i_{R_{\theta}} \omega_{2}^{\prime}, \bmod \theta$. Therefore

$$
i_{R_{\theta^{\prime}}}\left(\Pi_{1}^{*} \omega^{\prime}{ }_{1}-\Pi_{2}^{*} \omega_{2}^{\prime}\right) \equiv \frac{1}{\rho} i_{R_{\theta}}\left(\Pi_{1}^{*} \omega^{\prime}{ }_{1}-\Pi_{2}^{*} \omega^{\prime}{ }_{2}\right), \quad \bmod \theta
$$

Therefore $\mathcal{M}=\langle\theta, d \theta, \omega\rangle$ is independent of the choice of $\theta$. 
Suppose that $\left(E_{1}, E_{2}\right)$ is integrable. Let

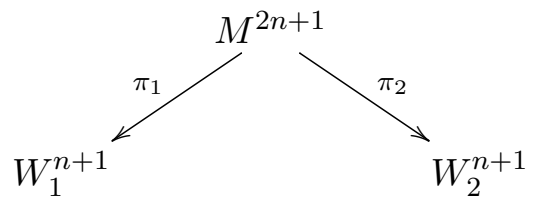

be a double Legendrian fibration induced by a Lagrangian pair $\left(E_{1}, E_{2}\right)$ locally. Suppose that a volume $(n+1)$-form $\Omega_{1}$ is given on $W_{1}$ (resp. a volume $(n+1)$-form $\Omega_{2}$ on $\left.W_{2}\right)$. Since $\left(T M / E_{2}\right)_{x} \cong T_{\pi_{1}(x)} W_{1}$ via $\pi_{1}$ (resp. $\left(T M / E_{1}\right)_{x} \cong T_{\pi_{2}(x)} W_{2}$ via $\left.\pi_{2}\right)$ for $x \in M, \Omega_{1}$ (resp. $\left.\Omega_{2}\right)$ is regarded as a non-zero section $\omega_{1}^{\prime}$ of $\wedge^{n+1}\left(T M / E_{1}\right)^{*}$ (resp. $\omega_{2}^{\prime}$ of $\left.\wedge^{n+1}\left(T M / E_{2}\right)^{*}\right)$. Then, following the general setting, we set

$$
\omega=i_{R_{\theta}}\left(\pi_{1}^{*} \Omega_{1}-\pi_{2}^{*} \Omega_{2}\right) .
$$

Note that $\pi_{1}^{*} \Omega_{1}, \pi_{2}^{*} \Omega_{2}$ are basic forms (cf. [14]) for $\pi_{1}, \pi_{2}$ respectively, however $i_{R_{\theta}} \pi_{1}^{*} \Omega_{1}$ and $i_{R_{\theta}} \pi_{2}^{*} \Omega_{2}$ need not to be basic.

Example 7.3. Consider $\mathbf{R}^{2 n+1}$ with coordinates $(x, z, p)=\left(x_{1}, \ldots, x_{n}, z, p_{1}, \ldots, p_{n}\right), D=\{\theta=$ $0\}, \theta=d z-\sum_{i=1}^{n} p_{i} d x_{i}, E_{1}^{\mathrm{st}}=\left\langle\frac{\partial}{\partial x_{1}}+p_{1} \frac{\partial}{\partial z}, \ldots, \frac{\partial}{\partial x_{n}}+p_{n} \frac{\partial}{\partial z}\right\rangle, E_{2}^{\mathrm{st}}=\left\langle\frac{\partial}{\partial p_{1}}, \ldots, \frac{\partial}{\partial p_{n}}\right\rangle, \pi_{1}: \mathbf{R}^{2 n+1} \rightarrow$ $\mathbf{R}^{n+1}, \pi_{1}(x, z, p)=(x, z), \pi_{2}: \mathbf{R}^{2 n+1} \rightarrow \mathbf{R}^{n+1}, \pi_{2}(x, z, p)=(p, x \cdot p-z)$. Set $\tilde{z}=x \cdot p-z$. The Reeb vector field for $\theta$ is given by $R=\frac{\partial}{\partial z}$.

Let $\pi_{1}^{*} \omega_{1}^{\prime}=f(x, z, p) d z \wedge d x_{1} \wedge \cdots \wedge d x_{n}$ be a non-zero section of $\wedge^{n+1}\left(T \mathbf{R}^{2 n+1} / E_{2}\right)^{*}$ pulledback to an $(n+1)$-form on $\mathbf{R}^{2 n+1}$. Then $i_{R}\left(\pi_{1}^{*} \omega_{1}^{\prime}\right)=f(x, z, p) d x_{1} \wedge \cdots \wedge d x_{n}$. Similarly, let $\pi_{2}^{*} \omega_{2}^{\prime}=-g(x, z, p) d \tilde{z} \wedge d p_{1} \wedge \cdots \wedge d p_{n}$ be a non-zero section of $\wedge^{n+1}\left(T \mathbf{R}^{2 n+1} / E_{1}\right)^{*}$ pulled-back to an $(n+1)$-form on $\mathbf{R}^{2 n+1}$. Then $i_{R}\left(\pi_{2}^{*} \omega_{2}^{\prime}\right)=g(x, z, p) d p_{1} \wedge \cdots \wedge d p_{n}$. Thus, following the general setting, we have

$$
\omega=f(x, z, p) d x_{1} \wedge \cdots \wedge d x_{n}-g(x, z, p) d p_{1} \wedge \cdots \wedge d p_{n},
$$

and we obtain Hesse Monge-Ampère systems.

Further, let $\Omega_{1}=f(x, z) d z \wedge d x_{1} \wedge \cdots \wedge d x_{n}\left(\right.$ resp. $\left.\Omega_{2}=-g(p, \tilde{z}) d \tilde{z} \wedge d p_{1} \wedge \cdots \wedge d p_{n}\right)$ be a volume form on $W_{1}=\mathbf{R}^{n+1}$ (resp. on $W_{2}=\mathbf{R}^{n+1}$ ). Then $i_{R} \pi_{1}^{*} \Omega_{1}=f(x, z) d x_{1} \wedge \cdots \wedge d x_{n}$ (resp. $\left.i_{R} \pi_{2}^{*} \Omega_{2}=g(p, \tilde{z}) d p_{1} \wedge \cdots \wedge d p_{n}\right)$. Thus we have

$$
\omega=f(x, z) d x_{1} \wedge \cdots \wedge d x_{n}-g(p, \tilde{z}) d p_{1} \wedge \cdots \wedge d p_{n},
$$

and we obtain Euler-Lagrange Monge-Ampère systems.

Remark 7.4. The Poincaré-Cartan form is given by $\Pi=\pi_{1}^{*} \Omega_{1}-\pi_{2}^{*} \Omega_{2}$ (see Remark 6.9).

\section{Homogeneous Monge-Ampère systems with Lagrangian pairs}

8.1. Monge-Ampère system on $\mathbf{R}^{2 n+1}$ as Hess $=c$ on $\mathbf{R}^{n+1}$. The Monge-Ampère system with Lagrangian pair for the equation $\operatorname{Hess}(f)=c$ in equi-affine geometry is given as follows.

Consider the contact manifold $M=\mathbf{R}^{2 n+1}$ with coordinates

$$
(x, z, p)=\left(x_{1}, \ldots, x_{n}, z, p_{1}, \ldots, p_{n}\right)
$$

and with the contact form $\theta=d z-\sum_{i=1}^{n} p_{i} d x_{i}$. We set two Lagrangian sub-bundles $E_{1}, E_{2}$ of the contact distribution $D=\{\theta=0\}$ by

$$
E_{1}=\left\langle\frac{\partial}{\partial x_{1}}+p_{1} \frac{\partial}{\partial z}, \ldots, \frac{\partial}{\partial x_{n}}+p_{n} \frac{\partial}{\partial z}\right\rangle, \quad E_{2}=\left\langle\frac{\partial}{\partial p_{1}}, \ldots, \frac{\partial}{\partial p_{n}}\right\rangle,
$$


which form a Lagrangian pair of $(M, D)$. The Reeb vector field $R$ is given by $\frac{\partial}{\partial z}$. Note that $-\theta=$ $d\left(\sum_{i=1}^{n} p_{i} x_{i}-z\right)-\sum_{i=1}^{n} x_{i} d p_{i}$. Then we have the double Legendrian fibration induced by $\left(E_{1}, E_{2}\right)$ :

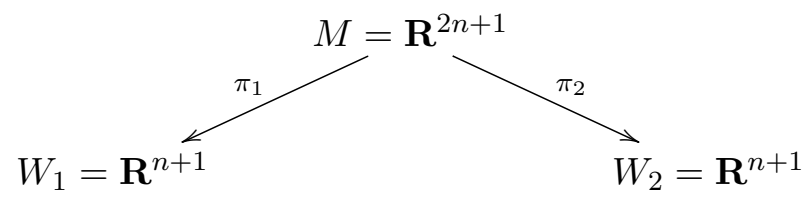

where

$$
\pi_{1}(x, z, p)=(x, z), \quad \pi_{2}(x, z, p)=(p, \tilde{z}), \quad \tilde{z}=\sum_{i=1}^{n} p_{i} x_{i}-z .
$$

Moreover take the $(n+1)$-forms

$$
\Omega_{1}=c\left(d z \wedge d x_{1} \wedge \cdots \wedge d x_{n}\right), \quad \Omega_{2}=-d \tilde{z} \wedge d p_{1} \wedge \cdots \wedge d p_{n}
$$

on $W_{1}=\mathbf{R}^{n+1}(c \in \mathbf{R}, c \neq 0)$ and on $W_{2}=\mathbf{R}^{n+1}$ respectively. Then $\omega=i_{R}\left(\pi_{1}^{*} \Omega_{1}-\pi_{2}^{*} \Omega_{2}\right)$ is given by

$$
\omega=c d x_{1} \wedge \cdots \wedge d x_{n}-d p_{1} \wedge \cdots \wedge d p_{n} .
$$

Thus we construct the Monge-Ampère system $\mathcal{M}=\langle\theta, d \theta, \omega\rangle$ with the Lagrangian pair $\left(E_{1}, E_{2}\right)$ globally on $M=\mathbf{R}^{2 n+1}$.

Proposition 8.1. Under the situation above, we have a Monge-Ampère system $\mathcal{M}$ generated by $(\theta, d \theta, \omega)$ with a Lagrangian pair $\left(E_{1}, E_{2}\right)$ on $M=\mathbf{R}^{2 n+1}$. The projection to $W_{1}=\mathbf{R}^{n+1}$ of a geometric solution of $\mathcal{M}$ satisfies the equation $\operatorname{Hess}(f)=c$, when it is represented as a graph $z=f\left(x_{1}, \ldots, x_{n}\right)$ outside of its singular locus. The projection to $W_{2}=\mathbf{R}^{n+1}$ of a geometric solution of $\mathcal{M}$ satisfies the equation $\operatorname{Hess}(f)=\frac{1}{c}$, when it is represented as a graph $z^{\prime}=\sum_{i=1}^{n} p_{i} x_{i}-z=f\left(p_{1}, \ldots, p_{n}\right)$ outside of its singular locus.

Remark 8.2. The Monge-Ampère system for Hess $=c$ is isomorphic to the system for Hess $=1$ if $c>0$, and is isomorphic to the system for Hess $=-1$ if $c<0$.

Remark 8.3. The Monge-Ampère system for Hess $=c, c \neq 0$ has the natural symmetry by the group $G^{\prime}$ of equi-affine transformations on $W_{1}=\mathbf{R}^{n+1}$ preserving the vector field $\frac{\partial}{\partial z}$. The group $G^{\prime}$ is given by the semi-direct product $G^{\prime}=G^{\prime \prime} \ltimes \mathbf{R}^{n+1}$ of $G^{\prime \prime} \subset \operatorname{SL}(n+1, \mathbf{R})$ and $\mathbf{R}^{n+1}$, where

$$
G^{\prime \prime}=\left\{\left(\begin{array}{cc}
A & 0 \\
t_{a} & 1
\end{array}\right) \mid A \in \mathrm{SL}(n, \mathbf{R}), a \in \mathbf{R}^{n}\right\} .
$$

Note that $\operatorname{dim} G^{\prime}=n(n+2)$ and also that each element of $G^{\prime}$ is identified with

$$
\left(\begin{array}{ccc}
1 & 0 & 0 \\
b & A & 0 \\
c & { }^{t} a & 1
\end{array}\right)
$$

via an appropriate embedding $\mathrm{SL}(n+1, \mathbf{R}) \hookrightarrow \mathrm{GL}(n+2, \mathbf{R})$. The Monge-Ampère system for Hess $=c, c \neq 0$, in fact, has bigger symmetry which attains the maximum for the dimension estimate of automorphisms given in Section 5 for bi-decomposable Monge-Ampère systems. 
Let $G$ be a subgroup of the projective transformation group $\operatorname{PGL}(n+2, \mathbf{R})$ on $\mathbf{R}^{n+2}$ consisting of transformations represented by the matrices

$$
\widetilde{A}=\left(\begin{array}{ccc}
\ell & 0 & 0 \\
b & A & 0 \\
c & { }^{t} a & k
\end{array}\right),
$$

considered up to non-zero scalar multiples. Here $\ell, k \in \mathbf{R}^{\times}, A \in \mathrm{GL}(n, \mathbf{R}), a, b \in \mathbf{R}^{n}, c \in \mathbf{R}$ satisfying the condition $(\operatorname{det} A)^{2}=(k \ell)^{n}$. From the condition, we can take $\operatorname{det} A= \pm 1, \ell=$ $\pm 1 / k$ in $\operatorname{PGL}(n+2, \mathbf{R})$. If $n$ is odd, then we can take $\operatorname{det} A=1$ and $\ell=1 / k$. Note that $\operatorname{dim} G=(n+1)^{2}$.

The group $G$ acts on $M=\mathbf{R}^{2 n+1}=\mathbf{R}^{n} \times \mathbf{R} \times \mathbf{R}^{n}$ transitively by

$$
\widetilde{A}(x, z, p)=\left(\frac{1}{\ell}(A x+b), \frac{1}{\ell}\left(k z-{ }^{t} a x-c\right), k\left(p-\frac{1}{k} t^{t} a\right) A^{-1}\right) .
$$

Here $a, b, x$ are regarded as column $n$-vectors and $p$ a row $n$-vector. The contact form $\theta=d z-p d x$ is transformed to $\frac{k}{\ell} \theta$, therefore the contact structure $D=\{\theta=0\}$ is $G$-invariant. The bidecomposable form $\omega$ is transformed to

$$
\frac{1}{\ell^{n}}(\operatorname{det} A) c\left(d x_{1} \wedge \cdots \wedge d x_{n}\right)-k^{n}(\operatorname{det} A)^{-1}\left(d p_{1} \wedge \cdots \wedge d p_{n}\right)=\frac{1}{\ell^{n}}(\operatorname{det} A) \omega,
$$

by the condition $(\operatorname{det} A)^{2}=(k \ell)^{n}$. Therefore $G$ leaves the Monge-Ampère system $\mathcal{M}=\langle\theta, d \theta, \omega\rangle$ for Hess $=c, c \neq 0$.

The group $G$ acts on $W_{1}=\mathbf{R}^{n+1}$ and on $W_{2}=\mathbf{R}^{n+1}$ respectively by

$$
\widetilde{A}(x, z)=\left(\frac{1}{\ell}(A x+b), \frac{1}{\ell}\left(k z-{ }^{t} a x-c\right)\right),
$$

and by

$$
\widetilde{A}(\tilde{z}, p)=\left(\frac{1}{\ell}\left(k \tilde{z}+k\left(p-\frac{1}{k} t a\right) A^{-1} b+c\right), k\left(p-\frac{1}{k} t a\right) A^{-1}\right) .
$$

Then the volume form $\Omega_{1}$ on $W_{1}$ (resp. $\Omega_{2}$ on $\left.W_{2}\right)$ is transformed to $\frac{k}{\ell^{n+1}}(\operatorname{det} A) \Omega_{1}$ (resp. $\left.\frac{k^{n+1}}{\ell}(\operatorname{det} A)^{-1} \Omega_{2}\right)$.

8.2. Monge-Ampère system on $T_{1} \mathbf{E}^{n+1}$ as $K=c$ in $\mathbf{E}^{n+1}$. In Sections 8.2-8.4, we describe the Monge-Ampère systems corresponding to the the equation of constant Gaussian curvature in Euclidean, spherical or hyperbolic geometry. To provide the concrete form of the Monge-Ampère system, we treat three cases separately.

In the famous paper of Gauss [26], the "Gaussian curvature" of a space surface is introduced as the ratio of areas in the "Gauss map" of the surface. We observe that the equation of constant Gaussian curvature is regarded as a Monge-Ampère system with Lagrangian pair as follows.

Consider the unit tangent bundle $T_{1} \mathbf{E}^{n+1}=\mathbf{E}^{n+1} \times S^{n}$ of the Euclidean space $\mathbf{E}^{n+1}$. The standard contact structure on $\mathbf{E}^{n+1} \times S^{n}$ is given by the one-form $\theta=y_{1} d x_{1}+y_{2} d x_{2}+\cdots+$ $y_{n+1} d x_{n+1}$ on $\mathbf{E}^{n+1} \times \mathbf{E}^{n+1}$, restricted to $\mathbf{E}^{n+1} \times S^{n}$. Here

$$
(x ; y)=\left(x_{1}, x_{2}, \ldots, x_{n+1} ; y_{1}, y_{2}, \ldots, y_{n+1}\right)
$$

is the system of coordinates on $\mathbf{E}^{n+1} \times \mathbf{E}^{n+1}$. We set the contact distribution $D=\{\theta=0\} \subset$ $T\left(\mathbf{E}^{n+1} \times S^{n}\right)$ and two Lagrangian subbundles of $D$ :

$$
\begin{aligned}
& E_{1}=\left\{u=\xi_{1} \frac{\partial}{\partial x_{1}}+\xi_{2} \frac{\partial}{\partial x_{2}}+\cdots+\xi_{n+1} \frac{\partial}{\partial x_{n+1}} \mid \xi_{1} y_{1}+\xi_{2} y_{2}+\cdots+\xi_{n+1} y_{n+1}=0\right\}, \\
& E_{2}=\left\{v=\eta_{1} \frac{\partial}{\partial y_{1}}+\eta_{2} \frac{\partial}{\partial y_{2}}+\cdots+\eta_{n+1} \frac{\partial}{\partial y_{n+1}} \mid v \text { is tangent to } S^{n}\right\},
\end{aligned}
$$


which form an integrable Lagrangian pair of $(M, D)$. Then we have the double Legendrian fibration induced by $\left(E_{1}, E_{2}\right)$ :

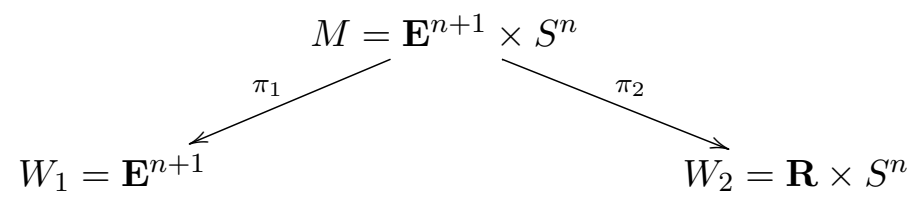

Here $\pi_{1}(x, y)=x, \pi_{2}(x, y)=(x \cdot y, y)$ for $(x, y) \in \mathbf{E}^{n+1} \times S^{n} \subset \mathbf{E}^{n+1} \times \mathbf{E}^{n+1}$.

Lemma 8.4. The above Lagrangian pair is flat, namely, the bi-Legendrian fibration is contactomorphic to the standard one: The Lagrangian pair $\left(E_{1}, E_{2}\right)=\left(\operatorname{Ker}\left(\pi_{2}\right)_{*}, \operatorname{Ker}\left(\pi_{1}\right)_{*}\right)$ is flat (see the standard example in Introduction).

Proof. On the open sets $U=\left\{(x, y) \in \mathbf{E}^{n+1} \times S^{n} \mid y_{n+1} \neq 0\right\}$ of $\mathbf{E}^{n+1} \times S^{n}$ and $V=\{(w, y) \in$ $\left.\mathbf{R} \times S^{n} \mid y_{n+1} \neq 0\right\}$ of $\mathbf{R} \times S^{n}$, take the system of coordinates $x_{i}^{\prime}=x_{i}, p_{i}^{\prime}=-\frac{y_{i}}{y_{n+1}}, z^{\prime}=x_{n+1}$, $1 \leq i \leq n$, on $U$, and define the diffeomorphism $\Phi: U \rightarrow \mathbf{R}^{2 n+1}$ by $\Phi(x, y)=\left(x^{\prime}, z^{\prime}, p^{\prime}\right)$. Moreover take the system of coordinates $\tilde{z}^{\prime}=\frac{w}{y_{n+1}}, p_{i}^{\prime}=-\frac{y_{i}}{y_{n+1}}, 1 \leq i \leq n$, on $V$ and define

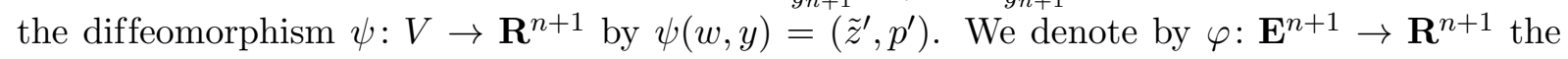
identity map, forgetting the Euclidean metric. Then $(\Phi, \varphi, \psi)$ induces the contactomorphism from $\left(U, D ; E_{1}, E_{2}\right)$ to $\left(\mathbf{R}^{2 n+1}, D_{\mathrm{st}} ; E_{1}^{\mathrm{st}}, E_{2}^{\mathrm{st}}\right)$. In fact $\theta=y_{n+1}\left(d z^{\prime}-\sum_{i=1}^{n} p_{i}^{\prime} d x_{i}^{\prime}\right)=y_{n+1}\left(\Phi^{-1}\right)^{*} \theta_{\mathrm{st}}$ and $y_{n+1}= \pm 1 / \sqrt{1+\sum_{i=1}^{n}\left(p_{i}^{\prime}\right)^{2}}$. Similarly on each open set $\left\{y_{i} \neq 0\right\}, 1 \leq i \leq n+1$, we see the flatness of $\left(E_{1}, E_{2}\right)$.

We endow $\mathbf{E}^{n+1}$ with the standard volume form

$$
\Omega_{1}=c d x_{1} \wedge d x_{2} \wedge \cdots \wedge d x_{n+1}
$$

multiplied with a real constant $c(\neq 0)$. Moreover we endow $\left(z ; y_{1}, y_{2}, \ldots, y_{n+1}\right) \in \mathbf{R} \times S^{n}$ $\left(\subset \mathbf{R} \times \mathbf{E}^{n+1}\right)$ with the standard volume form on $\mathbf{R} \times S^{n}$

$$
\Omega_{2}=\left.d z \wedge \sum_{i=1}^{n+1}\left((-1)^{i+1} y_{i} d y_{1} \wedge \cdots \wedge \breve{d y_{i}} \wedge \cdots \wedge d y_{n+1}\right)\right|_{\mathbf{R} \times S^{n}}
$$

The Reeb vector field $R$ on $\mathbf{E}^{n+1} \times S^{n}$ is given by $R=y_{1} \frac{\partial}{\partial x_{1}}+y_{2} \frac{\partial}{\partial x_{2}}+\cdots+y_{n+1} \frac{\partial}{\partial x_{n+1}}$, the "tautological" vector field. Then we set $\omega=i_{R}\left(\pi_{1}^{*} \Omega_{1}-\pi_{2}^{*} \Omega_{2}\right)$. Since

$$
\begin{aligned}
i_{R} \pi_{2}^{*} \Omega_{2} & =i_{R} d(x \cdot y) \wedge \sum_{i=1}^{n+1}\left((-1)^{i+1} y_{i} d y_{1} \wedge \cdots \wedge \breve{d y}_{i} \wedge \cdots \wedge d y_{n+1}\right) \\
& =\sum_{i=1}^{n+1}\left((-1)^{i+1} y_{i} d y_{1} \wedge \cdots \wedge \breve{d y_{i}} \wedge \cdots \wedge d y_{n+1}\right),
\end{aligned}
$$

we have

$$
\begin{aligned}
\omega= & c\left(y_{1} d x_{2} \wedge \cdots \wedge d x_{n+1}-y_{2} d x_{1} \wedge d x_{3} \wedge \cdots \wedge d x_{n+1}+\cdots+(-1)^{n} y_{n+1} d x_{1} \wedge \cdots \wedge d x_{n}\right) \\
& -\left(y_{1} d y_{2} \wedge \cdots \wedge d y_{n+1}-y_{2} d y_{1} \wedge d y_{3} \wedge \cdots \wedge d y_{n+1}+\cdots+(-1)^{n} y_{n+1} d y_{1} \wedge \cdots \wedge d y_{n}\right)
\end{aligned}
$$

on $\mathbf{E}^{n+1} \times S^{n}$.

This is exactly the reincarnation of the equation $K=c$ from the original definition due to Gauss. 
Proposition 8.5. Under the situation above, we have a Monge-Ampère system $\mathcal{M}$ generated by $(\theta, d \theta, \omega)$ with a Lagrangian pair $\left(E_{1}, E_{2}\right)$ on $M=T_{1} \mathbf{E}^{n+1}=\mathbf{E}^{n+1} \times S^{n}$. The Gaussian curvature of the projection to $W_{1}=\mathbf{E}^{n+1}$ of a geometric solution of $\mathcal{M}$ is equal to constant $c$ outside of singular locus.

Remark 8.6. The Monge-Ampère system for $K=c$ is isomorphic to the system for $K=1$ if $c>0$, and is isomorphic to the system for $K=-1$ if $c<0$.

Remark 8.7. Let $G$ be the Euclidean group on $\mathbf{E}^{n+1}$. The group $G$ acts on the orthonormal frame bundle transitively, therefore so on the unit tangent sphere bundle (the orthonormal 1Stiefel bundle) $\mathbf{E}^{n+1} \times S^{n}$ of $\mathbf{E}^{n+1}$. We fix the origin 0 in $\mathbf{E}^{n+1}$ and identify $\mathbf{E}^{n+1}$ with $\mathbf{R}^{n+1}$, giving the isomorphism $G \cong \mathrm{O}(n+1) \ltimes \mathbf{R}^{n+1}$. The contact structure $D=\{\theta=0\}$ and the contact form $\theta=\sum_{i=1}^{n+1} y_{i} d x_{i}$ on $\mathbf{E}^{n+1} \times S^{n}$ are $G$-invariant, and any $G$-invariant contact form is a non-zero multiple of $\theta$.

The group $G$ acts on $\mathbf{R} \times S^{n}$ by

$$
g(r, v)=(g(0) \cdot g(v)+r, g(v))=(b \cdot A v+r, A v),
$$

Here, for $g \in G$, we set $g(v)=A v+b\left(A \in \mathrm{O}(n+1), b \in \mathbf{R}^{n+1}\right)$. Then we get the diagram:

$$
\mathbf{E}^{n+1}=G / H^{\prime} \leftarrow \mathbf{E}^{n+1} \times S^{n}=G / H \rightarrow \mathbf{R} \times S^{n}=G / H^{\prime \prime},
$$

for the isotropy groups $H, H^{\prime}$ and $H^{\prime \prime}$ satisfying

$$
H^{\prime} \cong \mathrm{O}(n+1) \hookleftarrow H \cong \mathrm{O}(n) \hookrightarrow H^{\prime \prime} \cong \mathrm{O}(n) \ltimes \mathbf{R}^{n} .
$$

The $G$-invariant volume forms on $\mathbf{E}^{n+1}$ and $\mathbf{R} \times S^{n}$ are unique up to non-zero constant. We can construct the Monge-Ampère system $\mathcal{M}=\langle\theta, d \theta, \omega\rangle$ with the Lagrangian pair $\left(E_{1}, E_{2}\right)$ globally on $M=T_{1} \mathbf{E}^{n+1}$.

8.3. Monge-Ampère system on $T_{1} S^{n+1}$ as $K=c$ in $S^{n+1}$. Consider $\mathbf{E}^{n+2} \times \mathbf{E}^{n+2}$ with coordinates $x=\left(x_{0}, x_{1}, \ldots, x_{n+1}\right), y=\left(y_{0}, y_{1}, \ldots, y_{n+1}\right)$. Set $x \cdot y=\sum_{i=0}^{n+1} x_{i} y_{i}$, the standard inner product. Consider

$$
T_{1} S^{n+1}=\left\{(x, y) \in \mathbf{E}^{n+2} \times \mathbf{E}^{n+2}|| x|=1,| y \mid=1, x \cdot y=0\right\},
$$

the unit tangent bundle of $S^{n+1}$. The standard contact structure $D$ of $T_{1} S^{n+1}$ is defined by the contact form $\theta=\sum_{i=0}^{n+1} y_{i} d x_{i}$. Then we have the double Legendrian fibration

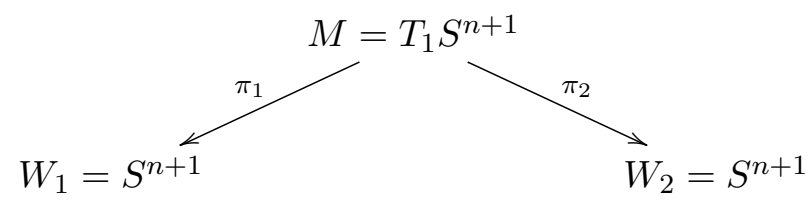

where $\pi_{1}(x, y)=x, \pi_{2}(x, y)=y$.

Lemma 8.8. The above bi-Legendrian fibration is contactomorphic to the standard model: The Lagrangian pair $\left(E_{1}, E_{2}\right)=\left(\operatorname{Ker}\left(\pi_{2}\right)_{*}, \operatorname{Ker}\left(\pi_{1}\right)_{*}\right)$ is flat. 
Proof. For instance, on the open subset $U=\left\{x_{0} \neq 0, y_{n+1} \neq 0\right\}$ of $T_{1} S^{n+1}$, consider the system of coordinates $x_{i}^{\prime}=\frac{x_{i}}{x_{0}}, z^{\prime}=-\frac{x_{n+1}}{x_{0}}, p_{i}^{\prime}=\frac{y_{i}}{y_{n+1}}, 1 \leq i \leq n$. Moreover we set $\tilde{z}^{\prime}=-\frac{y_{0}}{y_{n+1}}$. Then $\tilde{z}^{\prime}+z^{\prime}-\sum_{i=1}^{n} x_{i}^{\prime} p_{i}^{\prime}=0$ is satisfied. Moreover we have

$$
\theta=-x_{0} y_{n+1}\left(d z^{\prime}-\sum_{i=1}^{n} p_{i}^{\prime} d x_{i}^{\prime}\right)=x_{0} y_{n+1}\left(d \tilde{z}^{\prime}-\sum_{i=1}^{n} x_{i}^{\prime} d p_{i}^{\prime}\right)
$$

Thus we easily see that the diffeomorphism $\Phi: U \rightarrow \mathbf{R}^{2 n+1}$ defined by $\Phi(x, y)=\left(x^{\prime}, z^{\prime}, p^{\prime}\right)$ provides the required contactomorphism.

We set

$$
\Omega_{1}=c i_{X}\left(d x_{0} \wedge \cdots \wedge d x_{n+1}\right),
$$

the standard volume form on $W_{1}=S^{n+1}$ multiplied by $c(\neq 0) \in \mathbf{R}$, and set

$$
\Omega_{2}=i_{Y}\left(d y_{0} \wedge \cdots \wedge d y_{n+1}\right)
$$

the standard volume form on $W_{2}=S^{n+1}$. Here $X=\sum_{i=0}^{n+1} x_{i} \frac{\partial}{\partial x_{i}}$ and $Y=\sum_{i=0}^{n+1} y_{i} \frac{\partial}{\partial y_{i}}$. The Reeb vector field $R$ on $T_{1} S^{n+1}$ is given by $R=\sum_{i=0}^{n+1}\left(y_{i} \frac{\partial}{\partial x_{i}}-x_{i} \frac{\partial}{\partial y_{i}}\right)$. Then we have the following, which is the geometric form of the equation "Gaussian curvature $=c$ ".

Proposition 8.9. The associated Monge-Ampère system $\mathcal{M}$ with Lagrangian pair on $M=$ $T_{1} S^{n+1}$ is generated by $\theta$ and

$$
\begin{aligned}
& \omega=i_{R}\left(\pi_{1}^{*} \Omega_{1}-\pi_{2}^{*} \Omega_{2}\right) \\
& =c \sum_{0 \leq j<i \leq n+1}(-1)^{i+j} x_{i} y_{j} d x_{0} \wedge \cdots \wedge \breve{d x}_{j} \wedge \cdots \wedge \breve{d x_{i}} \wedge \cdots \wedge d x_{n+1} \\
& -c \sum_{0 \leq i<k \leq n+1}(-1)^{i+k} x_{i} y_{k} d x_{0} \wedge \cdots \wedge \breve{d x_{i}} \wedge \cdots \wedge \breve{d x}_{k} \wedge \cdots \wedge d x_{n+1} \\
& +\sum_{0 \leq j<i \leq n+1}(-1)^{i+j} y_{i} x_{j} d y_{0} \wedge \cdots \wedge \breve{d y}_{j} \wedge \cdots \wedge \breve{d y_{i}} \wedge \cdots \wedge d y_{n+1} \\
& -\sum_{0 \leq i<k \leq n+1}(-1)^{i+k} y_{i} x_{k} d y_{0} \wedge \cdots \wedge \breve{d y_{i}} \wedge \cdots \wedge \breve{d y}_{k} \wedge \cdots \wedge d y_{n+1} .
\end{aligned}
$$

The Gaussian curvature of the projection to $W_{1}=S^{n+1}$ of a geometric solution of $\mathcal{M}$ is equal to constant c outside of singular locus, while the Gaussian curvature of the projection to $W_{2}=S^{n+1}$ of a geometric solution of $\mathcal{M}$ is equal to constant $\frac{1}{c}$ outside of singular locus,

Remark 8.10. Note that the Gaussian curvature $K$ of a hypersurface in the unit sphere $S^{n+1}$ and its sectional curvature $S$ as a Riemannian manifold are related by $S=K+1$. For example, the great sphere $S^{n} \subset S^{n+1}$ has the constant Gauss map to $S^{n+1}$ and $K=0$, while has the sectional curvature 1 .

Remark 8.11. The group $G=\mathrm{O}(n+2)$ acts on $W_{1}=S^{n+1}$, on $W_{2}=S^{n+1}$ and on $S^{n+1} \times S^{n+1}$, thus on $T_{1} S^{n+1}=\left\{(x, y) \in S^{n+1} \times S^{n+1} \mid x \cdot y=0\right\}$. The contact structure $D=\{\theta=0\}$ and the contact form $\theta=\sum_{i=0}^{n+1} y_{i} d x_{i}$ are $G$-invariant, and any contact form defining $D$ is a non-zero constant multiple of $\theta$. 
We get the diagram:

$$
W_{1}=S^{n+1}=G / H^{\prime} \leftarrow M=T_{1} S^{n+1}=G / H \rightarrow W_{2}=S^{n+1}=G / H^{\prime \prime},
$$

for the isotropy groups $H, H^{\prime}$ and $H^{\prime \prime}$ satisfying

$$
H^{\prime} \cong \mathrm{O}(n+1) \hookleftarrow H \cong \mathrm{O}(n) \hookrightarrow H^{\prime \prime} \cong \mathrm{O}(n+1) .
$$

The $G$-invariant volume forms on $W_{1}=S^{n+1}$ and $W_{2}=S^{n+1}$ are unique up to non-zero constant. We can construct the Monge-Ampère system $\mathcal{M}=\langle\theta, d \theta, \omega\rangle$ with Lagrangian pair globally on $M=T_{1} S^{n+1}$.

8.4. Monge-Ampère system on $T_{1} H^{n+1}$ as $K=c$ in $H^{n+1}$. In general, let us consider $\mathbf{R}_{r}^{n+2}=\mathbf{R}^{n+2}$ with the inner product

$$
x \cdot y=-\sum_{i=0}^{r-1} x_{i} y_{i}+\sum_{j=r}^{n+1} x_{j} y_{j},
$$

of signature $(r, n+2-r)$. We set, for $\varepsilon_{1}=0, \pm 1, \varepsilon_{2}=0, \pm 1$, and for a real number $a$,

$$
S_{\varepsilon_{1}, \varepsilon_{2}, a}^{2 n+1}=\left\{(x, y) \in \mathbf{R}_{r}^{n+2} \times \mathbf{R}_{r}^{n+2} \mid x \cdot x=\varepsilon_{1}, y \cdot y=\varepsilon_{2}, x \cdot y=a, x \neq 0, y \neq 0\right\},
$$

provided that $S_{\varepsilon_{1}, \varepsilon_{2}, a}^{2 n+1} \neq \varnothing$. Moreover we set $S_{\varepsilon}^{n+1}=\left\{x \in \mathbf{R}_{r}^{n+2} \mid x \cdot x=\varepsilon, x \neq 0\right\}$ for $\varepsilon=0, \pm 1$. On $S_{\varepsilon_{1}, \varepsilon_{2}, a}^{2 n+1}$, the contact structure $D$ is defined by $\theta=-\sum_{i=0}^{r-1} y_{i} d x_{i}+\sum_{j=r}^{n+1} y_{j} d x_{j}$. We have the double Legendrian fibration



by $\pi_{1}(x, y)=x$ and $\pi_{2}(x, y)=y$.

In the case where $r=1, \varepsilon_{1}=-1, \varepsilon_{2}=1, a=0$, since

$$
\begin{aligned}
& S_{-1,1,0}^{2 n+1}=T_{1} H^{n+1}=T_{-1} S_{1}^{n+1} \subset \mathbf{R}_{1}^{n+2} \times \mathbf{R}_{1}^{n+2}, \\
& S_{-1}^{n+1}=H^{n+1}: \text { the hyperbolic space, } S_{1}^{n+1}: \text { the de Sitter space, }
\end{aligned}
$$

we have the double Legendrian fibration



(cf. the hyperbolic Gauss map [15]).

Lemma 8.12. The above bi-Legendrian fibration is contactomorphic to the standard model. The Lagrangian pair $\left(E_{1}, E_{2}\right)=\left(\operatorname{Ker}\left(\pi_{2}\right)_{*}, \operatorname{Ker}\left(\pi_{1}\right)_{*}\right)$ is flat.

Proof. For instance, on the open subset $\left\{x_{0} \neq 0, y_{n+1} \neq 0\right\}$, we take the system of coordinates $x_{i}^{\prime}=\frac{x_{i}}{x_{0}}, p_{i}^{\prime}=\frac{y_{i}}{y_{n+1}}, z^{\prime}=-\frac{x_{n+1}}{x_{0}}$. Moreover we set $\tilde{z}^{\prime}=\frac{y_{0}}{y_{n+1}}$. Then we have $z^{\prime}+\tilde{z}^{\prime}+\sum_{i=1}^{n} x_{i}^{\prime} p_{i}^{\prime}=0$, and $\theta=-x_{0} y_{n+1}\left(d z^{\prime}-\sum_{i=1}^{n} p_{i}^{\prime} d x_{i}^{\prime}\right)$. Then we see that the bi-Legendrian fibration is contactomorphic to the standard model. 
We set

$$
\Omega_{1}=c i_{X}\left(d x_{0} \wedge \cdots \wedge d x_{n+1}\right)
$$

the standard volume form on $W_{1}=H^{n+1}$ multiplied by $c \in \mathbf{R}(c \neq 0)$, and set

$$
\Omega_{2}=i_{Y}\left(d y_{0} \wedge \cdots \wedge d y_{n+1}\right)
$$

the standard volume form on $W_{2}=S_{1}^{n+1}$. Here $X=-x_{0} \frac{\partial}{\partial x_{0}}+\sum_{i=1}^{n+1} x_{i} \frac{\partial}{\partial x_{i}}$ and $Y=-y_{0} \frac{\partial}{\partial y_{0}}+$ $\sum_{i=1}^{n+1} y_{i} \frac{\partial}{\partial y_{i}}$. The Reeb vector field $R$ on $T_{1} H^{n+1}$ is given by $R=\sum_{i=0}^{n+1}\left(y_{i} \frac{\partial}{\partial x_{i}}+x_{i} \frac{\partial}{\partial y_{i}}\right)$. Then we have the following.

Proposition 8.13. The associated Monge-Ampère system $\mathcal{M}$ with Lagrangian pair on $M=$ $T_{1} H^{n+1}$ is generated by $\theta$ and

$$
\begin{aligned}
& \omega=i_{R}\left(\pi_{1}^{*} \Omega_{1}-\pi_{2}^{*} \Omega_{2}\right) \\
& =c \sum_{i=1}^{n+1}(-1)^{i} x_{0} y_{i} d x_{1} \wedge \cdots \wedge \breve{d x_{i}} \wedge \cdots \wedge d x_{n+1} \\
& +c \sum_{0 \leq j<i \leq n+1}(-1)^{i+j} x_{i} y_{j} d x_{0} \wedge \cdots \wedge \breve{d x}_{j} \wedge \cdots \wedge \breve{d x_{i}} \wedge \cdots \wedge d x_{n+1} \\
& -c \sum_{1 \leq i<k \leq n+1}(-1)^{i+k} x_{i} y_{k} d x_{0} \wedge \cdots \wedge \breve{d x}_{i} \wedge \cdots \wedge \breve{d x}_{k} \wedge \cdots \wedge d x_{n+1} \\
& +\sum_{i=1}^{n+1}(-1)^{i} y_{0} x_{i} d y_{1} \wedge \cdots \wedge \breve{d y_{i}} \wedge \cdots \wedge d y_{n+1} \\
& +\sum_{0 \leq j<i \leq n+1}(-1)^{i+j} y_{i} x_{j} d y_{0} \wedge \cdots \wedge \breve{d y}_{j} \wedge \cdots \wedge \breve{d y}_{i} \wedge \cdots \wedge d y_{n+1} \\
& -\sum_{1 \leq i<k \leq n+1}(-1)^{i+k} y_{i} x_{k} d y_{0} \wedge \cdots \wedge \breve{d y_{i}} \wedge \cdots \wedge \breve{d y_{k}} \wedge \cdots \wedge d y_{n+1} .
\end{aligned}
$$

The Gaussian curvature of the projection to $W_{1}=H^{n+1}$ of a geometric solution of $\mathcal{M}$ is equal to constant $c$ outside of singular locus.

Remark 8.14. The group $G=\mathrm{O}(1, n+1)$ acts on $W_{1}=H^{n+1}$, on $W_{2}=S_{1}^{n+1}$ and on $H^{n+1} \times S_{1}^{n+1}$, thus on $T_{1} H^{n+1}=\left\{(x, y) \in H^{n+1} \times S_{1}^{n+1} \mid x \cdot y=0\right\}$. The contact structure $D=\{\theta=0\}$ and the contact form $\theta=-y_{0} d x_{0}+\sum_{i=1}^{n+1} y_{i} d x_{i}$ are $G$-invariant, and any contact form defining $D$ is a non-zero constant multiple of $\theta$.

We get the diagram:

$$
W_{1}=H^{n+1}=G / H^{\prime} \leftarrow M=T_{1} H^{n+1}=G / H \rightarrow W_{2}=S_{1}^{n+1}=G / H^{\prime \prime},
$$

for the isotropy groups $H, H^{\prime}$ and $H^{\prime \prime}$ satisfying

$$
H^{\prime} \cong \mathrm{O}(n+1) \hookleftarrow H \cong \mathrm{O}(n) \hookrightarrow H^{\prime \prime} \cong \mathrm{O}(1, n)
$$

The $G$-invariant volume forms on $W_{1}=H^{n+1}$ and $W_{2}=S_{1}^{n+1}$ are unique up to non-zero constant. We can construct the Monge-Ampère system $\mathcal{M}=\langle\theta, d \theta, \omega\rangle$ with Lagrangian pair globally on $M=T_{1} H^{n+1}$. 
8.5. The Monge-Ampère systems introduced in Sections 8.1-8.4 are all Euler-Lagrange Monge-Ampère systems.

In fact, by Lemmas 8.4, 8.8, 8.12, there exists a system of local coordinates $\left(x^{\prime}, z^{\prime}, p^{\prime}\right)=$ $\left(x_{1}^{\prime}, \ldots, x_{n}^{\prime}, z^{\prime}, p_{1}^{\prime}, \ldots, p_{n}^{\prime}\right)$ at any point of $M$ such that a local contact form is given by $\theta=$ $d z^{\prime}-\sum_{i=1}^{n} p_{i}^{\prime} d x_{i}^{\prime}$, the Lagrangian pair is given by

$$
\begin{aligned}
& E_{1}=\left\{v \in T M \mid \theta(v)=0, d p_{1}^{\prime}(v)=0, \ldots, d p_{n}^{\prime}(v)=0\right\}, \\
& E_{2}=\left\{u \in T M \mid \theta(u)=0, d x_{1}^{\prime}(u)=0, \ldots, d x_{n}^{\prime}(u)=0\right\},
\end{aligned}
$$

the Monge-Ampère system $\mathcal{M}$ is generated by an $n$-form

$$
\tilde{\omega}=f\left(x_{1}^{\prime}, \ldots, x_{n}^{\prime}, z^{\prime}\right) d x_{1}^{\prime} \wedge \cdots \wedge d x_{n}^{\prime}-g\left(p_{1}^{\prime}, \ldots, p_{n}^{\prime}, \sum_{i=1}^{n} x_{i}^{\prime} p_{i}^{\prime}-z^{\prime}\right) d p_{1}^{\prime} \wedge \cdots \wedge d p_{n}^{\prime}
$$

for some (pulled-back) functions $f, g$ on $W_{1}, W_{2}$ respectively. If a local contactomorphism $\Phi: M \rightarrow \mathbf{R}^{2 n+1}$ and diffeomorphisms $\varphi: W_{1} \rightarrow \mathbf{R}^{n+1}, \psi: W_{2} \rightarrow \mathbf{R}^{n+1}$ give the contactomorphism of the bi-Legendrian fibration to the standard model, then we have

$$
\left(\varphi^{-1}\right)^{*} \Omega_{1}=f(x, z) d z \wedge d x_{1} \wedge \cdots \wedge d x_{n}, \quad\left(\psi^{-1}\right)^{*} \Omega_{2}=g(p, \tilde{z}) d \tilde{z} \wedge d p_{1} \wedge \cdots \wedge d p_{n},
$$

for some non-zero functions $f, g$.

For example, we calculate $f, g$ explicitly in Euclidean geometry (Section 8.2), for the system of coordinates $x_{i}^{\prime}=x_{i}, p_{i}^{\prime}=-y_{i} / y_{n+1}(1 \leq i \leq n), z^{\prime}=x_{n+1}$ on the open set $\left\{y_{n+1} \neq 0\right\}$. A direct calculation yields that the Monge-Ampère system for $K=c$ is generated by

$$
\tilde{\omega}=c d x_{1}^{\prime} \wedge \cdots \wedge d x_{n}^{\prime}-(-1)^{n}\left(1+p_{1}^{\prime 2}+\cdots+p_{n}^{\prime 2}\right)^{-\frac{n+2}{2}} d p_{1}^{\prime} \wedge \cdots \wedge d p_{n}^{\prime}
$$

with the contact form $\theta=d z^{\prime}-\sum_{i=1}^{n} p_{i}^{\prime} d x_{i}^{\prime}$.

Since $\left(\varphi^{-1}\right)^{*} \Omega_{1}$ and $\left(\psi^{-1}\right)^{*} \Omega_{2}$ are local volume forms on $\mathbf{R}^{n+1}$, the argument in Example 7.3 and Lemma 7.1 yields that all Monge-Ampère systems introduced in Sections 8.1-8.4 are EulerLagrange Monge-Ampère systems.

\section{Acknowledgements}

The first author was partially supported by Grants-in-Aid for Scientific Research No. 19654006 . The second author was partially supported by Grants-in-Aid for Scientific Research (C) No. 18540105. The authors would like to thank anonymous referees for the valuable comments to improve the paper.

\section{References}

[1] Alekseevsky D.V., Alonso-Blanco R., Manno G., Pugliese F., Contact geometry of multidimensional MongeAmpère equations: characteristics, intermediate integrals and solutions, Ann. Inst. Fourier (Grenoble) 62 (2012), 497-524, arXiv:1003.5177.

[2] Arnold V.I., Geometrical methods in the theory of ordinary differential equations, Grundlehren der Mathematischen Wissenschaften, Vol. 250, Springer-Verlag, New York - Berlin, 1983.

[3] Banos B., Nondegenerate Monge-Ampère structures in dimension 6, Lett. Math. Phys. 62 (2002), 1-15, math.DG/0211185.

[4] Banos B., On symplectic classification of effective 3-forms and Monge-Ampère equations, Differential Geom. Appl. 19 (2003), 147-166, math-ph/0003026. 
[5] Bryant R., Griffiths P., Grossman D., Exterior differential systems and Euler-Lagrange partial differential equations, Chicago Lectures in Mathematics, University of Chicago Press, Chicago, IL, 2003, math.DG/0207039.

[6] Bryant R.L., Bochner-Kähler metrics, J. Amer. Math. Soc. 14 (2001), 623-715, math.DG/0003099.

[7] Bryant R.L., Chern S.S., Gardner R.B., Goldschmidt H.L., Griffiths P.A., Exterior differential systems, Mathematical Sciences Research Institute Publications, Vol. 18, Springer-Verlag, New York, 1991.

[8] Gelfand I.M., Kapranov M.M., Zelevinsky A.V., Discriminants, resultants, and multidimensional determinants, Mathematics: Theory $\&$ Applications, Birkhäuser Boston, Inc., Boston, MA, 1994.

[9] Goursat E., Sur les équations du second ordre à $n$ variables analogues à l'équation de Monge-Ampère, Bull. Soc. Math. France 27 (1899), 1-34.

[10] Griffiths P., Harris J., Principles of algebraic geometry, Pure and Applied Mathematics, Wiley-Interscience, New York, 1978.

[11] Hitchin N., The geometry of three-forms in six dimensions, J. Differential Geom. 55 (2000), 547-576.

[12] Ishikawa G., Machida Y., Singularities of improper affine spheres and surfaces of constant Gaussian curvature, Internat. J. Math. 17 (2006), 269-293, math.DG/0502154.

[13] Ishikawa G., Morimoto T., Solution surfaces of Monge-Ampère equations, Differential Geom. Appl. 14 (2001), 113-124.

[14] Ivey T.A., Landsberg J.M., Cartan for beginners: differential geometry via moving frames and exterior differential systems, Graduate Studies in Mathematics, Vol. 61, Amer. Math. Soc., Providence, RI, 2003.

[15] Izumiya S., Pei D., Sano T., Singularities of hyperbolic Gauss maps, Proc. London Math. Soc. 86 (2003), $485-512$.

[16] Kobayashi S., Transformation groups in differential geometry, Ergebnisse der Mathematik und ihrer Grenzgebiete, Vol. 70, Springer-Verlag, New York - Heidelberg, 1972.

[17] Kobayashi S., Nomizu K., Foundations of differential geometry, Vol. II, Interscience Publishers, New York London, 1969.

[18] Kruglikov B., The D., The gap phenomenon in parabolic geometries, J. Reine Angew. Math., to appear, arXiv:1303.1307.

[19] Kushner A., Lychagin V., Rubtsov V., Contact geometry and nonlinear differential equations, Encyclopedia of Mathematics and its Applications, Vol. 101, Cambridge University Press, Cambridge, 2007.

[20] Lychagin V.V., Contact geometry and second-order nonlinear differential equations, Russ. Math. Surv. 34 (1979), 149-180.

[21] Lychagin V.V., Rubtsov V.N., Chekalov I.V., A classification of Monge-Ampère equations, Ann. Sci. École Norm. Sup. 26 (1993), 281-308.

[22] Machida Y., Morimoto T., On decomposable Monge-Ampère equations, Lobachevskii J. Math. 3 (1999), $185-196$.

[23] Morimoto T., La géométrie des équations de Monge-Ampère, C. R. Acad. Sci. Paris Sér. A-B 289 (1979), A25-A28.

[24] Morimoto T., Monge-Ampère equations viewed from contact geometry, in Symplectic Singularities and Geometry of Gauge Fields, Banach Center Publ., Vol. 39, Polish Acad. Sci. Inst. Math., Warsaw, 1998, 105-120.

[25] Nomizu K., Sasaki T., Affine differential geometry. Geometry of affine immersions, Cambridge Tracts in Mathematics, Vol. 111, Cambridge University Press, Cambridge, 1994.

[26] Spivak M., A comprehensive introduction to differential geometry. Vol. II, 2nd ed., Publish or Perish, Inc., Wilmington, Del., 1979.

[27] Tabachnikov S., Geometry of Lagrangian and Legendrian 2-web, Differential Geom. Appl. 3 (1993), 265-284.

[28] Takeuchi M., Lagrangean contact structures on projective cotangent bundles, Osaka J. Math. 31 (1994), $837-860$.

[29] Tanaka N., On generalized graded Lie algebras and geometric structures. I, J. Math. Soc. Japan 19 (1967), $215-254$.

[30] Tanaka N., On the equivalence problems associated with simple graded Lie algebras, Hokkaido Math. J. 8 (1979), 23-84.

[31] Yamaguchi K., Differential systems associated with simple graded Lie algebras, in Progress in Differential Geometry, Adv. Stud. Pure Math., Vol. 22, Math. Soc. Japan, Tokyo, 1993, 413-494. 